Experimentally Measured Total $X$-Ray Attenuation Coefficients Extracted from Previously Unprocessed Documents Held by the NIST Photon and Charged Particle Data Center
John H. Hubbell

U.S. DEPARTMENT OF COMMERCE Technology Administration National Institute of Standards and Technology Physics Laboratory

lonizing Radiation Division

Gaithersburg, MD 20899

$Q C$

100

.056

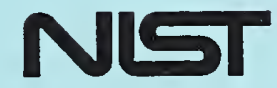

N0.5893 

Experimentally Measured Total $X$-Ray Attenuation Coefficients

Extracted from Previously

Unprocessed Documents Held

by the NIST Photon and Charged

Particle Data Center
John H. Hubbell

U.S. DEPARTMENT OF COMMERCE Technology Administration National Institute of Standards and Technology

Physics Laboratory

lonizing Radiation Division

Gaithersburg, MD 20899

September 1996

Prepared for:

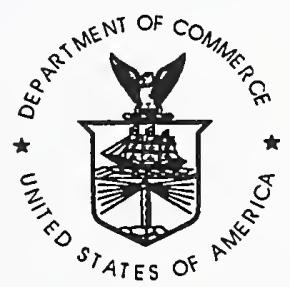

U.S. DEPARTMENT OF COMMERCE Michael Kantor, Secretary

TECHNOLOGY ADMINISTRATION

Mary L. Good, Under Secretary for Technology

NATIONAL INSTITUTE OF STANDARDS

AND TECHNOLOGY

Arati Prabhakar, Director 



\title{
Experimentally Measured Total X-Ray Attenuation Coefficients Extracted from Previously Unprocessed Documents Held by the NIST Photon and Charged Particle Data Center
}

\author{
J. H. Hubbell ${ }^{+}$ \\ 11830 Rocking Horse Road, Rockville, MD 20852
}

\begin{abstract}
This report lists and annotates 47 previously unprocessed original-source documents held by the NIST Photon and Charged Particle Data Center (PCPDC), containing experimentally measured absolute values of the mass attenuation coefficient, or total photon interaction cross section for one or more elemental materials. The data from these documents had not been previously extracted for purposes of entering into the PCPDC x-ray attenuation measurement data base. Tables of the extracted data, with all photon energies converted to multiples of eV (electron volts), and all photon cross sections converted to units of barns/atom, are given. A total of 3735 data points were extracted and are presented.
\end{abstract}

Key words: attenuation coefficient, cross section, data base, gamma ray, photon, $\mathrm{x}$ ray

\footnotetext{
*Work supported by the Office of Standard Reference Data of the National Institute of Standards and Technology.
}

${ }^{+}$Work carried out for the National Institute of Standards and Technology under contract 43NANB513350. 



\section{Introduction}

Since 1950 the National Institute of Standards and Technology (NIST; formerly National Bureau of Standards) has maintained a data base of measured and theoretical x-ray attenuation and cross section data in a hard-copy file of original source documents in the form of reprints, reports, and personal communications. This data base extends over 11 decades of photon energies, from $10 \mathrm{eV}$ (extreme ultraviolet or XUV region) to $100 \mathrm{GeV}$ (cosmic-ray energy region). The purpose of this data base, now residing in the NIST Photon and Charged Particle Data Center (PCPDC), is to provide photon (XUV, X-ray, gamma-ray, and bremsstrahlung) interaction data required in a variety of medical, industrial, defense, and basic-science applications. This NIST/PCPDC data base, unique in the global scientific community, has been used from time to time as the basis for the tabulation of photon cross sections and attenuation coefficients [1-15] and related quantities such as the photon energy-transfer and energy-absorption coefficients $[5-7,9,11,16-18]$.

In collaboration with $\mathrm{H}$. Gerstenberg, E. Saloman and a series of summer student assistants, the data extracted from the accumulated NIST/PCPDC-held documents (1907-1986) were converted to a machine-readable form in 1986 [19-24]. Since 1986, additional measurements have been made and reported in the literature. Also, some additional earlier documents, dating back to 1981 , have been acquired by the Center. It is the purpose of this report to extract and put in machine-readable and standardized form the data from these additional documents 1981-1995, which are listed and annotated in Section V.

\section{Procedures}

In the above hard-copy file of original source documents, the measurements were made in a great variety of contexts and scientific disciplines and for different purposes. These purposes range from medical x-ray therapy and diagnostic applications to x-ray crystallography, to aircraft radiometric fuel gauge development, and to many other extremely diverse human endeavors requiring reliable values of $x$-ray interaction data. Hence, the measured results in these source documents were given by the authors in units peculiar to the particular scientific discipline in which the measurements were taken.

For example, in much of the x-ray crystallography and x-ray fluorescence (XRF) analytical literature, as also in much of the atomic physics basic research literature, the photon energies are not given, but rather the photon wavelengths, in units of angstroms $(\AA)$ or in nanometers $(\mathrm{nm})$. The photon interaction probabilities (cross sections) are also given in a variety of different forms, such as: linear attenuation coefficients (e.g., $\mathrm{cm}^{-1}$ ), mass attenuation (or absorption) coefficients (e.g., $\mathrm{cm}^{2} / \mathrm{g}$ or $\mathrm{m}^{2} / \mathrm{kg}$ ), cross sections (e.g., b/atom [barns/atom], or $\mathrm{Mb} / \mathrm{atom}$, or $\mathrm{Mb} / \mathrm{molecule}$ for binary gases, etc.). In some documents the data are not given numerically, but only graphically. In these cases, if the author could not provide a numerical listing of the data, the numerical values were read from the published graphs. In this report, all photon energies (or wavelengths) are converted to energies in units of eV, and all totalinteraction cross section or attenuation coefficient data points are converted to units of b/atom. 
Many of the documents also include data for compounds and mixtures of elements. However, except for mono-element binary gases such as $\mathrm{H}_{2}, \mathrm{~N}_{2}$ and $\mathrm{O}_{2}$, this report is restricted to elements only.

\section{Results}

The extracted photon attenuation data are given in Table 1, in terms of the total interaction cross section in b/atom as a function of photon energy in electron volts $(\mathrm{eV})$. The data in Table 1 are grouped by element and arranged in ascending order of the atomic number $\mathrm{Z}$. Within the group for each element, the data sets from the individual papers are arranged in ascending order of the lowest photon energy for which a data point is given in that data set. The number of data points is indicated, also the reference symbol derived from the year of publication and the first two letters of the first author's last name, with an additional number attached as a suffix to ensure uniqueness.

Following the precedent set by Saloman et al., in references [20] and [23], all photon-energy and cross-section data points arc rounded off to four significant figures, and are given in scientific notation with the power of ten multiplier as a suffix.

As can be seen in Table 1, the number data points for a given element in a given original source document varies greatly. For example, in the paper by Ye Zongyuan et al. [84Ye01], the measurements were all taken at a single photon energy $(7.28 \mathrm{MeV})$, resulting in single-point sets for the elements $\mathrm{Mg}, \mathrm{Al}, \mathrm{Fe}, \mathrm{Ni}, \mathrm{Cd}, \mathrm{Sn}$, and $\mathrm{Bi}$. At the other extreme is the set of 458 data points measured for Xe by Deutsch et al. [91De01] in their examination of the Xe K-edge over the narrow range of photon energies 33.0 to $40.6 \mathrm{keV}$.

Table 1 is summarized in Table 2, showing for each element the number of papers which contain data for that element, and the total number of data points extracted from these papers. From the 47 papers examined, for all elements, a grand total of 3735 data points were extracted.

Authors' estimates of uncertainty, if provided, are noted in the square brackets at the end of each annotated referen - in the document listing in Section V. These stated uncertainties vary greatly, fro $\pm 0.2 \%$ to $\pm 50 \%$. In general, a more meaningful "envelope of uncertainty" is obtained by noting differences between independent measurements for the same element and energies by different authors under different experimental conditions. 


\section{Text References}

1. White (Grodstein), G.R., X-Ray Attenuation Coefficients from $10 \mathrm{kev}$ to $100 \mathrm{MeV}$, NBS Rep. 1003 (1952).

2. Fano, U., Gamma-Ray Attenuation, Part 1, Nucleonics 11 (8), 8-12 (1953); Part 2, 11 (9), 55-60 (1953).

3. Grodstein, G.R., X-ray Attenuation Coefficients from $10 \mathrm{kev}$ to $100 \mathrm{MeV}$, NBS Circ. 583 (1957).

4. McGinnies, R.T., X-Ray Attenuation Coefficients from $10 \mathrm{keV}$ to $100 \mathrm{MeV}$, Suppl. to NBS Circ. 583 (1959).

5. Berger (McGinnies), R.T., The X- or Gamma-Ray Energy Absorption or Transfer Coefficient: Tabulations and Discussion, Radiat. Res. 15, 1-29 (1961).

6. Hubbell, J.H. and Berger, M.J., Sec. 4.1: Attenuation Coefficients, Energy Absorption Coefficients, and Related Quantities (p. 167-184) and Sec. 4.2: Photon Atomic Cross Sections (p. 185-202). IAEA Engineering Compendium on Radiation Shielding, R.G. Jaeger, ed., Springer, Berlin (1968).

7. Hubbell, J.H., Photon Cross Sections, Attenuation Coefficients, and Energy Absorption Coefficients from $10 \mathrm{keV}$ to $100 \mathrm{GeV}$, NSRDS-NBS Rep. 29 (1969).

8. Hubbell, J.H, McMaster, W.H., Del Grande, N.K., and Mallett, J.H., Sec. 2.1: X-Ray Cross Sections and Attenuation Coefficients (p. 47-70), IUCr International Tables for $X$-Ray Crystallography, J.A. Ibers and W.C. Hamilton, eds., Kynoch Press, Birmingham, England (1974); based on McMaster, W.H., Del Grande, N.K., Mallett, J.H., and Hubbell, J.H., Compilation of X-Ray Cross Sections, Lawrence Livermore National Laboratory Report UCRL-50174, Sec II, Rev.1 (1969).

9. Hubbell, J.H., Photon Mass Attenuation and Mass Energy-Absorption Coefficients for H, $\mathrm{C}, \mathrm{N}, \mathrm{O}, \mathrm{Ar}$, and Seven Mixtures from $0.1 \mathrm{keV}$ to $20 \mathrm{MeV}$, Radiat. Res. 70, 58- 81 (1977).

10. Hubbell, J.H., Gimm, H.A. and Øverbø, I., Pair, Triplet and Total Atomic Cross Sections (and Mass Attenuation Coefficients) for $1 \mathrm{MeV}-100 \mathrm{GeV}$ Photons in Elements $\mathrm{Z}=1$ to 100, J. Phys. Chem. Ref. Data 9, 1023-1147 (1980).

11. Hubbell, J.H., Photon Mass Attenuation and Energy-Absorption Coefficients from $1 \mathrm{keV}$ to $20 \mathrm{MeV}$, Int. J. Appl. Radiat. Isot. 33, 1269-1290 (1982).

12. Berger, M.J. and Hubbell, J.H., XCOM: Photon Cross Sections on a Personal Computer, National Bureau of Standards Internal Report NBSIR 87-3597 (1987).

13. Cullen, D.E., Chen, M.H., Hubbell, J.H., Perkins, S.T., Plechaty, E.F., Rathkopf. J.A. and Scofield, J.H., Tables and Graphs of Photon-Interaction Cross Sections from $10 \mathrm{eV}$ to $100 \mathrm{GeV}$ Derived from the LLNL Evaluated Photon Data Library. Part A: Z $=1$ to 50. Part B: $Z=51$ to 100 , Lawrence Livermore National Laboratory Report $\mathrm{UCRL}=50400$, Vol. 6, Rev. 4 (1989). 
14. Creagh, D.C. and Hubbell, J.H., X-Ray Absorption (or Attenuation) Coefficients, Section 4.2.4 in IUCr International Tables for Crystallography, Vol. C, A.J.C. Wilson, Editor, Kluwer Academic Publishers (Dordrecht/Boston/London 1992), 189-206.

15. Berger, M.J. and Hubbell, J.H., Photon Attenuation Coefficients, in CRC Handbook of Chemistry and Physics, 76th Edition, D.R. Lide, Editor-in-Chief (CRC Press, Inc., 19951996), $10-284$ to $10-288$.

16. Higgins, P.D., Attix, F.H., Hubbell, J.H., Seltzer, S.M., Berger, M.J. and Sibata, C.H., Mass Energy-Transfer and Mass Energy-Absorption Coefficients, Including In-Flight Positron Annihilation for Photon Energies $1 \mathrm{keV}$ to $100 \mathrm{MeV}$, National Institute of Standards and Technology Internal Report NISTIR 4812 (1992).

17. Seltzer, S.M., Calculation of Photon Energy-Transfer and Mass Energy-Absorption Coefficients, Rad. Res. 136, 147-170 (1993).

18. Seltzer, S.M. and Hubbell, J.H., Tables and Graphs of Photon Mass Attenuation Coefficients and Mass Energy Absorption Coefficients for Photon Energies $1 \mathrm{keV}$ to $20 \mathrm{MeV}$ for Elements $\mathrm{Z}=1$ to 92 and Some Dosimetric Materials, Japanese Society of Radiological Technology Report ISSN 1340-7716 (1995); see also Hubbell. J.H. and Seltzer, S.M., National Institute of Standards and Technology Report NISTIR 5632 (1995).

19. Gerstenberg, H.M., and Hubbell, J.H., Comparison of Experimental with Theoretical Photon Attenuation Cross Sections Between $10 \mathrm{eV}$ and $100 \mathrm{GeV}$ (p. 1007-1009), Nuclear Data for Science and Technology, K.H. Bockhoff, ed., ECSC, EEC, EAEC, Brussels and Luxembourg (1983).

20. Saloman, E.B., and Hubbell, J.H., X-Ray Attenuation Coefficients (Total Cross Sections): Comparison of the Experimental Data Base with the Recommended Values of Henke and the Theoretical Values of Scofield for Energies Between 0.1-100 keV, National Bureau of Standards Internal Report NBSIR 86-3431 (1986).

21. Hubbell, J.H., Gerstenberg, H.M. and Saloman, E.B., Bibliography of Photon Total Cross Section (Attenuation Coefficient) Measurements $10 \mathrm{eV}$ to $13.5 \mathrm{GeV}$, National Bureau of Standards Internal Report NBSIR 86-3461 (1986).

22. Saloman, E.B., and Hubbell, J.H., Critical Analysis of Soft X-Ray Cross Section Data, (Presented at the Third International Symposium on Radiation Physics, Ferrara, Italy, Sept. 30-Oct. 4, 1985), Nucl. Instrum. Meth. A 255, 38-42 (1987).

23. Saloman, E.B., Hubbell, J.H. and Scofield, J.H., X-Ray Attenuation Cross Sections for Energies $100 \mathrm{eV}$ to $100 \mathrm{keV}$ and Elements $\mathrm{Z}=1$ to $\mathrm{Z}=92$, Atomic Data and Nuclear Data Tables 38, 1-197 (1988).

24. Saloman, E.B., Hubbell, J.H. and Berger, M.J., National Bureau of Standards Data Base of Photon Absorption Cross Sections from $10 \mathrm{eV}$ to $100 \mathrm{GeV}$, Proc. SPIE 911, 100-106 (1988). 
V. Annotated List of Documents from which Data Were Extracted

(Authors' estimates of uncertainty $[ \pm \%]$, where given.)

$81 \mathrm{Wu02} \mathrm{Wu}$, C.Y.R. and Judge, D. L., J. Chem. Phys. 75, 172-178 (1981); also personal communication to J.H. Hubbell from Wu 5/22/86; same data in Wu, C.Y.R., J. Chem. Phys. 77, 1179-1182 (1982)

Lyman- $\alpha$ Fluorescence from Hydrogen Photofragments of $\mathrm{CH}_{4}$ and $\mathrm{H}_{2} \mathrm{O}$ $\left(19-69 \mathrm{eV}: \mathrm{N}\right.$ [in $\mathrm{N}_{2}$ ], $\mathrm{O}$ [in $\mathrm{O}_{2}$ ], $\mathrm{CO}, \mathrm{NO}, \mathrm{CO}_{2}, \mathrm{~N}_{2} \mathrm{O}, \mathrm{CH}_{4}, \mathrm{C}_{2} \mathrm{H}_{4}, \mathrm{C}_{2} \mathrm{H}_{6}, \mathrm{C}_{2} \mathrm{H}_{10}$ ) [ $\pm 30 \%]$

82A101 Alaverdov, V. I. and Podolyak, E. R., Opt. Spectrosc. (USSR) 53, 665-666 (1982); transl. from Opt. Spektrosk. 53, 1113-1114 (1982)

Photoabsorption Cross Section for Molecular Hydrogen in the 70-200-eV Region (72.4-192.6 eV: $\mathrm{H}$ [in $\mathrm{H}_{2}$ ]) [ $\pm 2.0 \%$ to $4.3 \%$ ]

82Me01 Mehlman, G., Cooper, J. W. and Saloman, E. B., Phys. Rev. A 25, 2113-2122 (1982) Absolute Photoabsorption Cross Section of the K Shell of Atomic Lithium $(68.9-103.3 \mathrm{eV}: \mathrm{Li})[ \pm 20 \%]$

82Tu01 Tuilier, M. H., Laporte, D. and Esteva, J. M., Phys. Rev. A 26, 372-378 (1982); cross sections (graphical) given in: M.-H. Tuilier: Photoabsorption du Sodium Atomique dans la Region du Seuil d'Ionisation 1s, Ph.D. Thesis, Univ. P. et Marie Curie, Paris (1983) (1.071-1.091 keV: $\mathrm{Na})$ [ $\pm 50 \%]$

84Na01 Nathuram, R., Rao, I.S.S., and Mehta, M. K., Indian J. Phys. A 58, 300-304 (1984)

Experimental Mass Absorption Coefficients for Low Energy Photons in Beryllium, Carbon, Aluminum, Silicon, Copper, and Lead $(13.432-59.20 \mathrm{keV}$ : $\mathrm{Be}, \mathrm{C}, \mathrm{Al}, \mathrm{Si}, \mathrm{Cu}, \mathrm{Pb})[ \pm 1 \%$ to $2 \%]$

84Ye01 Ye Zongyuan, Li Jingwen, Jin Zheng, Sun Zhongfa and Shi Detang, Chinese J. Nucl. Phys. 6, 334-341 (1984) (in Chinese; English transl. in NIST [J. Hubbell] file) Total Photon Cross Section Measurements for some Metals at $\mathrm{E}_{\gamma}=7.28 \mathrm{MeV}$ (7.28 $\mathrm{MeV}: \mathrm{Mg}, \mathrm{Al}, \mathrm{Fe}, \mathrm{Ni}, \mathrm{Cd}, \mathrm{Sn}, \mathrm{Bi})[ \pm 6.4 \%$ to $12.2 \%]$

86De01 Deutsch, M. and Hart, M., J. Phys. B 19, L303-L307 (1986); also personal communication to J. Hubbell with numerical data $\mathrm{X}$-Ray Attenuation Cross Section Measurements in the K Edge Region of Krypton (14.24638-14.74454 keV: Kr)

86De02 Del Grande, N. K., SPIE (Int. Soc. Optical Engineering) Proc. Vol. 691, 2-10 (1986) Measured 1 to $40 \mathrm{keV}$ Photoabsorption Cross Sections for: $\mathrm{Fe}, \mathrm{Ni}, \mathrm{Sn}, \mathrm{Ta}, \mathrm{Pt}, \mathrm{Au}, \mathrm{Pb}, \mathrm{U}$ (1.000-40.00 keV: Fe, Ni, Sn, Ta, Pt, Au, Pb, U) [ $\pm 3 \%]$

87De01 Del Grande, N. K., Tirsell, K. G., Schneider, M. B., Garrett, R. F., Kneedler, E. M. and Manson, S. T., J. Physique 48, Colloque C9, 951-954 (1987) Resonances in Near-Threshold X-Ray Photoabsorption of Inner Shells (80-400 eV: C, U) [ $\pm 10 \%]$

87De02 Del Grande, N. K., Presented at Workshop on New Directions in Soft X-Ray Near Threshold Phenomena, Pacific Grove, Calif. March 1-4, 1987, UCRL-96162 (1987) Anomalies in Near-Threshold Photoabsorption: Resonances, "White Lines" and Other Enhancements

(80-1000 eV: C, Ti, Cr, Ni, Cu, Th, U) [ $\pm 10 \%]$ 
87Ke01 Kefi, M., Andre, J. M. and Bonnelle, C., J. Physique 48, Colloque C9, 907-910 (1987) X-Ray Attenuation Measurements of Medium-Z Elements Around their K-Edge

Data in M. Kefi Ph. D. Thesis (Univ. Pierre et Marie Curie, Paris), Attenuation Coefficients and Atomic Scattering Factors of the Elements 45 to 54 in the Region of their $\mathrm{K}$ Absorption Edge

(16-44 keV: Pd, Ag, Cd, In, Sn, I, Xe) [ $\pm 2.0 \%$ to $3.2 \%]$

87Ma01 Machali, F., Al-Barakati, G. G., El-Sayed, A. A. and Altaf, W. J., J. Phys. F 17, 1279-1284 (1987)

The Photoelectric Cross Section of Gamma Rays in the Energy Range 43 to $152 \mathrm{keV}$ (43-152 keV: $\mathrm{Al}, \mathrm{Cu}, \mathrm{Zn}, \mathrm{Ag}, \mathrm{Pt}, \mathrm{Au}, \mathrm{Pb})[ \pm 0.8 \%$ to $1.3 \%]$

87Re01 Reddy, B. S., Rao, P.V.R., Premchand, K. and Parthasaradhi, K., Phys. Rev. A 35, 1430-1432 (1987)

Photon Cross Sections in $\mathrm{Cu}, \mathrm{Pt}$, and $\mathrm{Au}$ at $81 \mathrm{keV}$

(79.623-80.905 keV: $\mathrm{Cu}, \mathrm{Pt}, \mathrm{Au})[ \pm 1.3 \%$ to $3.1 \%]$

87Sa01 Sarma, P. R., Lakshminarayana, V., Parthasaradhi, K., Narasimham, K. L. and Reddy, S. B., Nucl. Instrum. Meth. A 257, 378-380 (1987)

Precision Measurements of Total Gamma Ray Cross Sections with a High Resolution Detector

(93.613-136.474 keV: Pd, Cd, Er, Pt) [ $\pm 1 \%]$

87Sa02 Sasi, P. R., Lakshminarayana, V., Parthasaradhi, K., Ramaniah, K. V., Raju, G. K. and Narasimham, K. L., J. Phys. B 20, 2641-2645 (1987)

Total Gamma Ray Cross Section Measurements in Pd, Pt and Tungsten-Steel Alloy Using a High-Resolution Detector

(109.78-383.86 keV: Pd, Pt, tungsten-steel) [士1\%]

87Ya01 Yang, B. X. and Kirz, J., Applied Optics 26, 3823-3826 (1987)

Soft X-Ray Absorption Cross Section of Argon Determined by a Variable Absorber

Technique

(0.326-1.01 keV: Ar) [ $\pm 1.5 \%]$

88An01 Angel, G. C. and Samson, J.A.R., Phys. Rev. A 38, 5578-5585 (1988)

Total Photoionization Cross Sections of Atomic Oxygen from Threshold to $44.3 \AA$ (13.6-280 eV: O) [ $\pm 9 \%]$

88Na01 Nathuram, R., Rao, I.S.S. and Mehta, M. K., Phys. Rev. A 37, 4978-4981 (1988) Photoelectric Cross Sections for 6-20 -keV Photons in Beryllium, Carbon, Magnesium, Aluminum, Silicon, Copper, Silver, and Lead

(5.89-20.16 keV: Be, C, Mg, Al, Si, Cu, Ag, Pb) [ $\pm 1 \%]$

88Pa01 Parthasaradhi, K., Esposito, A., Mobilio, S. and Pelliccioni, M., Phys. Rev. A 38, 1608-1611 (1988)

Photon Cross Sections Near the X-Ray-Absorption Edges of $\mathrm{Ti}, \mathrm{Ni}, \mathrm{Pt}$, and $\mathrm{Au}$ (4.966-12.320 keV: Ti, Ni, Pt, Au) [ $\pm 3 \%$ to 4\%]

89Ge01 Gerward, L., Acta Cryst. A 45, 1-3 (1989)

$\mathrm{X}$-Ray Attenuation Coefficients of Beryllium in the Energy Range 5 to $20 \mathrm{keV}$ (5.415-17.479 keV: Be) [ $\pm 1 \%$ to $2 \%]$ 
89Ke01 Kerur, B. R., Jahagirdar, H. A., Thontadarya, S. R. and Hanumaiah, B., Am. J. Phys. 57, 1148-1149 (1989)

(52.4 keV: Al, Ni, Cu, Ag, Sn, Pb)

89Sa01 Samson, J.A.R. and Yin, L., J. Optical Soc. Am. B 6, 2326-2333 (1989)

Precision Measurements of Photoabsorption Cross Sections of $\mathrm{Ar}, \mathrm{Kr}, \mathrm{Xe}$, and Selected Molecules at 58.4, 73.6, and $74.4 \mathrm{~nm}$

(16.7-21.2 eV: $\mathrm{H}_{2}, \mathrm{~N}_{2}, \mathrm{O}_{2}, \mathrm{Ar}, \mathrm{Kr}, \mathrm{Xe}, \mathrm{CH}_{4}, \mathrm{CO}, \mathrm{CO}_{2}, \mathrm{~N}_{2} \mathrm{O}$ ) [ $\left.\pm 0.8 \%\right]$

89Un01 Unonius, L. and Suortti, P., J. Appl. Cryst. 22, 46-52 (1989)

Mass Attenuation Coefficients of the Elements $\mathrm{Ti}, \mathrm{V}, \mathrm{Fe}, \mathrm{Co}, \mathrm{Ni}, \mathrm{Cu}$, and $\mathrm{Zn}$ for the $\mathrm{K}$ Emission Lines Between 4.51 and $10.98 \mathrm{keV}$

(4.509-10.982 keV: Ti, V, Fe, Co, Ni, Cu, Zn) [ $\pm 1 \%]$

90De01 Del Grande, N. K., Physica Scripta 41, 110-114 (1990)

L Shell Photoabsorption Spectroscopy for Solid Metals: Ti, V, Cr, Fe, Ni, Cu

(0.40-1.50 keV: Ti, Cr, Fe, Ni, Cu, [V not measured]) [ $\pm 10 \%$ ]

90Fu01 Fujisaki, H., Nakagiri, N., Nagata, H., Kihara, N. and Miyahara, T., Japanese J. Appl. Phys. 29, 1357-1361 (1990); also personal communication to J. Hubbell with numerical data

Measurement of Soft X-Ray Absorption by Al, Cr, and Ni Using Synchrotron Radiation (234.3-989.2 eV: $\mathrm{Al}, \mathrm{Cr}, \mathrm{Ni})[ \pm 4 \%$ to $50 \%]$

90Qu01 Quamruzzaman, K., Hussain, M. and Sarkar, M., Dhaka University Studies B 38, 155-160 (1990)

Measurement of the Total X-Ray Attenuation Cross Sections of Al, Ti, Fe, Zn, Cd, Sn and $\mathrm{W}$ at $22.6 \mathrm{keV}$

(22.6 keV: Al, Ti, Fe, $\mathrm{Zn}, \mathrm{Cd}, \mathrm{Sn}, \mathrm{W})[ \pm 2 \%$ to $3 \%]$

91De01 Deutsch, M., Brill, G. and Kizler, P., Phys, Rev. A 43, 2591-2594 (1991); also personal communication to J. Hubbell with numerical data Near-K-Edge Photoabsorption Measurements in Xenon

(33.00-40.60 keV: Xe)

91El01 El-Kateb, A. H. and Abdul-Hamid, A. S., Appl. Radiat. Isot. 42, 303-307 (1991) Photon Attenuation Coefficient Study of Some Materials Containing Hydrogen, Carbon and Oxygen (54.-1333. $\mathrm{keV}: \mathrm{H}, \mathrm{C}, \mathrm{O})[ \pm 2 \%$ to $11 \%]$

91Ke01 Kerur, B. R., Thontadarya, S. R. and Hanumaiah, B., Appl. Radiat. Isot. 42, 571-575 (1991)

A Novel Method for the Determination of X-Ray Mass Attenuation Coefficients (5.947 keV: Al, Cu, Ag, Lucite, Mylar, Nylon, Teflon) [ $\pm 1 \%$ ]

92Ja01 Jahagirdar, H. A., Hanumaiah, B. and Thontadarya, S. R., Appl. Radiat. Isot. 43, 399-404 (1992)

A New Direct Method to Determine Total Atomic Photoelectric Cross Sections at $123.6 \mathrm{keV}$

(123.6 keV: $\mathrm{Ag}, \mathrm{Pb})[ \pm 0.3 \%$ to $10 \%]$ 
92Ja02 Jahagirdar, H. A., Hanumaiah, B. and Thontadarya, S. R., Appl. Radiat. Isot. 43, $1511-1514$ (1992)

Determination of Narrow Beam Attenuation Coefficients from a Broad Beam Geometrical Configuration for $320 \mathrm{keV}$ Photons

(320 keV: $\left.\mathrm{Pb}, \mathrm{BiPO}_{4},\left(\mathrm{CH}_{3} \mathrm{COO}\right)_{2} \cdot \mathrm{Pb} \cdot 3 \mathrm{H}_{2} \mathrm{O}, \mathrm{Na}_{2} \mathrm{WO}_{4} \cdot 2 \mathrm{H}_{2} \mathrm{O}, \mathrm{Pb}\left(\mathrm{NO}_{3}\right)_{2}\right)[ \pm 3 \%]$

92Ke02 Kerur, B. R., Thontadarya, S. R and Hanumaiah, B., X-Ray Spectrom. 21, 133-136 (1992)

Effect of Photon Intensity Selected at Various Positions Under the Photopeak on the Mass Attenuation Coefficient of X-Rays

$(5.947,6.46 \mathrm{keV}: \mathrm{Al})[ \pm 1 \%]$

92Wa01 Wang Dachun, Ding Xunliang, Wang Xinfu, Yang Hua, Zhou Hongyu, Shen Xinyin and Zhu Guanghua, Nucl. Instrum. Meth. B 71, 241-248 (1992); also see 91 Wa01 $\mathrm{X}$-Ray Attenuation Coefficients and Photoelectric Cross Sections of $\mathrm{Cu}$ and $\mathrm{Fe}$ in the Range $3 \mathrm{keV}$ to $29 \mathrm{keV}$

(3.313-29.109 keV: $\mathrm{Fe}, \mathrm{Cu})[ \pm 1 \%]$

92Wa02 Wang Dachun, Yang Hua, Luo Pingan and Ding Xunliang, Nucl. Instrum. Meth. B 71, 249-254 (1992)

Measurements of Mass Attenuation Coefficients of Yttrium with Characteristic Lines from Elements Excited by Energetic Protons

(3.313-29.109 keV: Y) [ $\pm 1 \%]$

93Ja01 Jahagirdar, H. A., Hanumaiah, B. and Thontadarya, S. R., Appl. Radiat. Isot. 44, 1248-1252 (1993)

Measurement of Narrow Beam Attenuation Coefficients Using a Broad Beam Geometry Configuration for $145.4 \mathrm{keV}$ Photons

(145.4 keV: $\mathrm{Pb}, \mathrm{BiPO}_{4},\left(\mathrm{CH}_{3} \mathrm{COO}\right)_{2} \cdot \mathrm{Pb} \cdot 3 \mathrm{H}_{2} \mathrm{O}, \mathrm{Na}_{2} \mathrm{WO}_{4} \cdot 2 \mathrm{H}_{2} \mathrm{O}, \mathrm{Pb}\left(\mathrm{NO}_{3}\right)_{2}, \mathrm{PbO}$, $\left.\mathrm{PbO}_{2}\right)[ \pm 1 \%]$

93Ke01 Kerur, B. R., Thontadarya, S. R. and Hanumaiah, B., X-Ray Spectrom. 22, 13-16 (1993)

X-Ray Attenuation Coefficients at $6.46 \mathrm{keV}$ and the Validity of the Mixture Rule for Compounds

(6.46 keV: Al, Cu, Mo, Ta, Nylon, PTFE, Lucite, $\mathrm{CaSO}_{4} \cdot 2 \mathrm{H}_{2} \mathrm{O}, \mathrm{Co}\left(\mathrm{C}_{2} \mathrm{H}_{3} \mathrm{O}_{2}\right)_{2} \cdot 4 \mathrm{H}_{2} \mathrm{O}$, $\left.\mathrm{Cr}\left(\mathrm{C}_{2} \mathrm{H}_{3} \mathrm{O}_{2}\right)_{3} \cdot \mathrm{H}_{2} \mathrm{O}, \mathrm{MgSO}_{4} \cdot 7 \mathrm{H}_{2} \mathrm{O}, \mathrm{ZnSO}_{4} \cdot 7 \mathrm{H}_{2} \mathrm{O}\right)[ \pm 0.2 \%$ to $0.8 \%]$

93Ke02 Kerur, B. R., Thontadarya, S. R. and Hanumaiah, B., X-Ray Spectrom. 22, 156-159 (1993)

Measurement of X-Ray Attenuation Coefficients Around K-Absorption Edges Using Fe $\mathrm{K}_{\alpha} \mathrm{X}$-Rays

(6.400 keV: $\mathrm{Al}, \mathrm{Cu}, \mathrm{Mo}, \mathrm{Ta}, \mathrm{CaSO}_{4} \cdot 2 \mathrm{H}_{2} \mathrm{O}, \mathrm{Co}\left(\mathrm{C}_{2} \mathrm{H}_{3} \mathrm{O}_{2}\right)_{2} \cdot 4 \mathrm{H}_{2} \mathrm{O}, \mathrm{Cr}\left(\mathrm{C}_{2} \mathrm{H}_{3} \mathrm{O}_{2}\right)_{3} \cdot \mathrm{H}_{2} \mathrm{O}$, $\left.\mathrm{FeSO}_{4} \cdot 7 \mathrm{H}_{2} \mathrm{O}, \mathrm{MgSO}_{4} \cdot 7 \mathrm{H}_{2} \mathrm{O}, \mathrm{MnSO}_{4} \cdot \mathrm{H}_{2} \mathrm{O}, \mathrm{NaVO}_{3}, \mathrm{ZnSO}_{4} \cdot 7 \mathrm{H}_{2} \mathrm{O}\right)[ \pm 1.8 \%]$

93Ke03 Kerur, B. R., Thontadarya, S. R. and Hanumaiah, B., Indian J. Phys. A 67, 163-167 (1993)

Measurement of X-Ray Mass Attenuation Coefficients Using Mixed Radiation Emitters $(6.46,8.118 \mathrm{keV}: \mathrm{Al}, \mathrm{Cu}, \mathrm{Ta}$, Nylon, Lucite, Teflon) $[ \pm 0.2 \%$ to $0.8 \%]$ 
93Na01 Nair, K.P.G., Gowda, C., Kumari, J. S., Anasuya, S. J., Umesh, T. K. and Gowda, R., Nucl. Sci. Engin. 115, 300-303 (1993)

Total Interaction Cross Sections of Several Sugars for ${ }^{133} \mathrm{Ba}$ Photons

(30.8-384.0 keV: Al, Cu, arabinose, fructose, galactose, glucose, maltose, mannose, melezitose, melibiose, raffinose, rhamnose, ribose) [ $\pm 2 \%]$

94Gu01 Gullikson, E. M., Denham, P., Mrowka, S. and Underwood, J. H., Phys. Rev. B 49, 16283-16288 (1994)

Absolute Photoabsorption Measurements of $\mathrm{Mg}, \mathrm{Al}$, and $\mathrm{Si}$ in the Soft X-Ray Region Below the $\mathrm{L}_{2,3}$ Edges

(25-97 eV: Mg, Al, Si)

94Ke01 Kerur, B. R., Thontadarya, S. R. and Hanumaiah, B., Appl. Radiat. Isot. 45, 159-163 (1994)

Anomalous X-Ray Attenuation Coefficients Around the Absorption Edges Using $\mathrm{Mn} \mathrm{K}_{\alpha}$ and $\mathrm{Cu} \mathrm{K} \mathrm{K}_{\alpha} \mathrm{X}$-Rays

$(5.895,8.041 \mathrm{keV}: \mathrm{Al}, \mathrm{Cu}, \mathrm{Ta})[ \pm 0.4 \%$ to $1.0 \%]$

94Sa01 Samson, J.A.R., He, Z. X., Yin, L. and Haddad, G. N., J. Phys. B 27, 887-898 (1994)

Precision Measurements of the Absolute Photoionization Cross Sections of He

$(24.6-118.0 \mathrm{eV}: \mathrm{He})[ \pm 1 \%$ to $2 \%]$

94Wa01 Wang Dachun, Yang Hua and Luo Ping-an, Nucl. Instr. Meth. B 86, 231-235 (1994)

Measurements of the Mass Attenuation Coefficients of Tin with Characteristic Lines from Elements Excited by Energetic Protons

(3.313-15.165 keV: Sn) [ $\pm 1 \%]$

94Wa02 Wang Dachun, Yang Hua and Wang Xin-Min, Nucl. Instr. Meth. B 86, 236-240 (1994)

Mass Attenuation Coefficients of Indium in the Energy Range 2.6 to $29.1 \mathrm{keV}$

(2.622-29.11 keV: In) $[ \pm 1 \%]$

95Az01 Azuma, Y., Berry, H. G., Gemmell, D. S., Suleiman, J., Westerlind, M., Sellin, I. A., Woicik, J. C. and Kirkland, J. P., Phys. Rev. A 51, 447-453 (1995)

Attenuation of Photons at $3-14-\mathrm{keV}$ Energies in Helium

(3.35-14.00 $\mathrm{keV}: \mathrm{He})[ \pm 1 \%$ to $2 \%]$

95Ta01 Tajuddin, A. A., Chong, C. S., Shukri, A., Bandyopadhyay, T. and Bradley, D. A., Appl. Radiat. Isot. 46, 113-115 (1995)

Measurement of Mass Attenuation Coefficients of Moderate-to-High Atomic-Number Elements at Low Photon Energies

(13.9-59.5 keV: Cu, Zr, Nb, Mo, Pd, Ag, Cd, In, Sn, Hf, Ta, W, Re) [ $\pm 3 \%$ to $12 \%$ ]

95Wa01 Wang Dachun, Luo Ping-An and Yang Hua, Nucl. Instr. Meth. B 95, 161-165 (1995)

Measurement of the Mass Attenuation Coefficients for $\mathrm{SiH}_{4}$ and $\mathrm{Si}$

(1.486-29.109 $\left.\mathrm{keV}(\mathrm{Si}): \mathrm{SiH}_{4}, \mathrm{Si}\right)[ \pm 1 \%$ to $2 \%]$ 


\section{Table 1. Experimental Total Interaction Cross Section Data Extracted from Documents}

$\mathrm{Z}=1, \mathrm{H}\left(\mathrm{in} \mathrm{H}_{2}\right)$

$1.667+014.745+06$

$\mathrm{Z}=1, \mathrm{H}\left(\mathrm{in} \mathrm{H}_{2}\right)$

$7.240+017.175+04$

1. $140+021 \cdot 599+04$

$\mathrm{Z}=1, \mathrm{H}$

$5.400+046.410-01$

5. $110+05 \quad 3.314-01$

1. $333+061.841-01$

$\mathrm{Z}=2, \mathrm{He}$

$2.459+017.400+06$

$2.800+016.050+06$

3. $200+014.820+06$

3. $600+013.880+06$

$4.000+013.160+06$

$4.400+012.600+06$

$4.800+012.190+06$

$5.200+011.850+06$

$5.600+011.630+06$

$6.019+015.500+05$

$6.520+011.200+06$

$6.800+011.060+06$

$7.200+019.070+05$

$7.600+017.880+05$

$8.000+016.930+05$

$8.400+016.140+05$

$8.800+015.460+05$

$9.200+014.880+05$

9. $600+014.380+05$

$1.000+023.930+05$

$1.040+023.550+05$

$1.080+023.220+05$

$1.120+022.920+05$

$1.160+022.660+05$

$\mathrm{Z}=2, \mathrm{He}$

$3.350+039.750+00$

$4.150+035.260+00$

$4.950+033.550+00$

$5.550+032.930+00$

$8.000+031.840+00$

1. $200+041.480+00$

$Z=3$, Li (vapor)

$6.890+012.800+06$

$8.260+012.100+06$

$\mathrm{Z}=4, \mathrm{Be}$

$5.415+034.938+01$

$8.905+031.259+01$

$\mathrm{Z}=4, \mathrm{Be}$

$5.890+033.750+01$

$\mathrm{Z}=4, \mathrm{Be}$

1. $343+045 \cdot 103+00$

$5.920+041.901+00$

eV, b/atom pairs

3 pts

$\begin{array}{llll}1.685+01 & 5.175+06 & 2.122+01 & 3.235+06\end{array}$

eV, b/atom pairs

8 pts

$7.530+016.184+04 \quad 8.120+015.265+04$

$1.328+029.940+03 \quad 1.511+026.755+03$

$\mathrm{eV}, \mathrm{b} / \mathrm{atom}$ pairs

9 pts

$8.100+04 \quad 5.155-01 \quad 1.230+054.703-01$

$6.620+05 \quad 2.561-01 \quad 1.173+06 \quad 1.958-01$

$\mathrm{eV}, \mathrm{b} / \mathrm{atom}$ pairs

$2.500+017.210+06$

$2.900+015.700+06$

$3.300+014.570+06$

$3.700+013.680+06$

$4.100+013.010+06$

$4.500+012.480+06$

$4.900+012.100+06$

$5.700+011.610+06$

$6.287+011.220+06$

$6.540+011.190+06$

$6.900+011.020+06$

$7.300+018.730+05$

$7.700+017.620+05$

$8.100+016.720+05$

$8.500+015.950+05$

$8.900+015.310+05$

$9.300+014.750+05$

$9.700+01 \quad 4.270+05$

$1.010+023.830+05$

$1.050+023.450+05$

$1.090+023.130+05$

$1.130+022.860+05$

1. $170+02 \quad 2.610+05$
$5.300+011.770+06$

2. $600+0195$ pts

$2.600+016.790+06$

3. $000+015.380+06$

3. $400+014.320+06$

3. $800+013.500+06$

$4.200+012.860+06$

$4.600+012.380+06$

$5.000+012.020+06$

$5.400+011.710+06$

$5.800+011.580+06$

$6.431+011.170+06$

$6.600+011.150+06$

$7.000+019.750+05$

$7.400+018.420+05$

$7.800+017.380+05$

$8.200+016.520+05$

$8.600+015.780+05$

9. $000+015.160+05$

$9.400+014.640+05$

$9.800+014.160+05$

1. $020+023.730+05$

$1.060+023.380+05$

$1.100+023.060+05$

1. $140+022.790+05$

$1.180+022.550+05$

$\mathrm{eV}, \mathrm{b} / \mathrm{atom}$ pairs

$3.550+038.070+00$

$4.350+03 \quad 4.660+00$

$5.150+033.300+00$

$6.000+032.570+00$

$9.000+031.700+00$

1. $300+041.470+00$

23 pts

$3.750+036.930+00$

$4.550+034.160+00$

$5.350+033.110+00$

$6.500+032.410+00$

1. $000+041.610+00$

$1.400+041 \cdot 410+00$

ev, b/atom pairs

$7.210+012.400+06 \quad 7.510+012.700+06$

$8.860+01 \quad 1.750+06 \quad 9.540+01 \quad 1.600+06$

$\mathrm{eV}, \mathrm{b} / \mathrm{atom}$ pairs

6 pts

$6.404+03 \quad 3.008+01 \quad 6.930+03 \quad 2.409+01$

$1.749+043.816+00$

ev, b/atom pairs

4 pts

$1.337+04 \quad 4.900+00 \quad 1.744+04 \quad 3.900+00$

$\mathrm{eV}, \mathrm{b} /$ atom pairs

5 pts

$\begin{array}{llll}1.722+04 & 3.861+00 & 2.016+04 & 3,367+00\end{array}$ ref $=89 \mathrm{Sa01}$

ref $=82 \mathrm{Al} 01$

$9.640+012.729+04$

$1.926+023.231+03$

ref $=91 \mathrm{E} 101$

3. $560+053.532-01$

1.274+06 1.875-01

ref $=94 \mathrm{SaOl}$

$2.700+016.400+06$

$3.100+015.100+06$

$3.500+014.090+06$

$3.900+013.320+06$

$4.300+012.720+06$

$4.700+012.280+06$

$5.100+011.940+06$

$5.500+011.670+06$

$5.900+011.560+06$

$6.474+011.100+06$

$6.700+011.100+06$

$7.100+019.400+05$

$7.500+018.130+05$

$7.900+017.150+05$

$8.300+016.330+05$

$8.700+015.610+05$

$9.100+015.020+05$

$9.500+014.500+05$

$9.900+014.050+05$

$1.030+023.630+05$

$1.070+023.280+05$

$1.110+023.000+05$

$1.150+022.730+05$

ref $=95 \mathrm{Az} 01$

$3.950+036.050+00$

$4.750+03 \quad 3.790+00$

$5.500+03 \quad 3.070+00$

$7.000+032.140+00$

1. $100+041.560+00$

ref $=82 \mathrm{Me} 01$

$7.750+012.400+06$

$1.033+021.220+06$

ref $=89 \mathrm{Ge} 01$

$8.048+031.616+01$

ref $=88 \mathrm{NaOl}$

$2.016+043.400+00$

ref $=84 \mathrm{NaOI}$

$4.652+042.305+00$ 
$\mathrm{Z}=6, \mathrm{C}$

$2.000+021.300+05$

$2.400+029.000+04$

2. $750+021.000+05$

$2.900+021.020+06$

$3.000+021.300+06$

$3.200+028.300+05$

3. $600+026.400+05$

$4.000+024.500+05$

$\mathrm{Z}=6, \mathrm{C}$

$5.890+032.330+02$

$\mathrm{Z}=6, \mathrm{C}$

$4.652+043.949+00$

$\mathrm{Z}=6, \mathrm{C}$

$5.400+043.630+00$

$5.110+051.715+00$

$1.333+061.117+00$

$\mathrm{Z}=7, \mathrm{~N}\left(\right.$ in $\left.\mathrm{N}_{2}\right)$

1. $667+019.970+06$

$\mathrm{Z}=7, \quad \mathrm{~N}\left(\right.$ in $\left.\mathrm{N}_{2}\right)$

1.907+01.1.165+07

$2.033+01 \quad 1.155+07$

$2.175+011.185+07$

$2.339+011.280+07$

$2.530+011.085+07$

$2.755+011.100+07$

$3.024+011.030+07$

$3.351+019.050+06$

$3.757+017.000+06$

$4.275+015.450+06$

$4.959+014.700+06$

$5.904+013.550+06$

\section{$\mathrm{Z}=8,0$}

$1.361+011.500+05$

$1.366+012.750+06$

1. $393+012.700+06$

1. $442+013.100+06$

$1.476+013.300+06$

$1.579+013.700+06$

$1.632+013.900+06$

$1.701+019.170+06$

$1.722+019.000+06$

$1.736+019.180+06$

1. $748+019.710+06$

$1.783+019.060+06$

$1.745+019.470+06$

$1.816+019.690+06$

1. $907+011.300+07$

$2.033+011.340+07$

$2.175+01$ 1.290+07

$2.339+011.210+07$

$2.530+011.200+07$

3. $100+011.180+07$

$3.444+019.570+06$

3. $875+018.250+06$

4. $275+017.400+06$

eV, b/atom pairs

29 pts

2. $100+02$ 1.200+05

$2.500+028.000+04$

$2.780+021.200+05$

$2.920+021.250+06$

3. $050+021.140+06$

$3.300+027.300+05$

3. $700+026.000+05$

2. $200+021.100+05$

$2.600+028.000+04$

$2.800+022.450+04$

2. $950+021.300+06$

3. $100+029.600+05$

$3.400+027.200+05$

$3.800+025.500+05$

eV, b/atom pairs

4 pts

$\begin{array}{llll}1.337+04 & 2.190+01 & 1.744+04 & 1.190+01\end{array}$

$\mathrm{eV}, \mathrm{b} / \mathrm{atom}$ pairs

2 pts

$5.920+043.490+00$

$\mathrm{eV}, \mathrm{b} / \mathrm{atom}$ pairs

9 pts

$8.100+04 \quad 3.211+00 \quad 1.230+05 \quad 2.872+00$

$6.620+051.536+00 \quad 1.173+06 \quad 1.177+00$

eV, b/atom pairs

3 pts

$\begin{array}{llll}1.685+01 & 8.430+06 & 2.122+01 & 1.139+07\end{array}$

eV, b/atom pairs

48 pts

$1.937+01 \quad 1.165+07 \quad 1.968+011.160+07$

$2.066+01 \quad 1.155+07$

2. $214+011.210+07$

$2.384+01 \quad 1.250+07$

$2.583+011.085+07$

$2.818+011.095+07$

3. $100+011.000+07$

3. $444+018.650+06$

$3.875+016.400+06$

$4.428+015.200+06$

5. $166+014.500+06$

$6.199+013.200+06$

$2.101+011.165+07$

$2.254+01 \quad 1.255+07$

$2.431+01 \quad 1.135+07$

$2.638+011.085+07$

$2.883+01 \quad 1.090+07$

$3.179+019.700+06$

3. $542+016.200+06$

$4.000+016.000+06$

$4.592+015.100+06$

$5.391+014.200+06$

$6.526+012.900+06$

$\mathrm{eV}, \mathrm{b} /$ atom pairs

1. $362+017.000+05$

$1.370+012.750+06$

$1.401+012.850+06$

$1.450+013.100+06$

$1.485+013.300+06$

$1.590+013.700+06$

$1.648+014.150+06$

$1.704+018.900+06$

$1.724+019.000+06$

$1.740+019.260+06$

$1.752+019.880+06$

$1.787+019.000+06$

$1.797+019.800+06$

$1.830+01 \quad 1.000+07$

$1.937+01 \quad 1.330+07$

$2.066+01 \quad 1.330+07$

2. $214+01$ 1.270+07

$2.384+01 \quad 1.200+07$

$1.883+01 \quad 1.150+07$

3. $179+01$ 1.050+07

3. $542+019.250+06$

$4.000+017.900+06$

$4.428+016.850+06$

\section{7 pts}

1. $362+011.150+06$ $1.378+012.850+06$ $1.425+013.100+06$

$1.459+013.200+06$

$1.494+013.300+06$

$1.621+01 \quad 4.000+06$

$1.693+018.030+06$

$1.716+019.020+06$

$1.728+018.790+06$

$1.744+019.300+06$

$1.756+019.710=06$

$1.790+019.600+06$

1. $813+019.250+06$

$1.864+01 \quad 1.200+07$

$1.968+01 \quad 1 \cdot 340+07$

$2.101+01 \quad 1.320+07$

$2.254+01 \quad 1.250+07$

$2.431+011.190+07$

$2.952+01 \quad 1.130+07$

3. $263+01 \quad 1.020+07$

$3.647+018.920+06$

$4.081+017.700+06$

$4.592+016.500+06$ ref $=87 \mathrm{De} 01$

$2.300+029.000+04$

$2.700+029.000+04$

$2.850+027.800+05$

2. $970+021.330+06$

3. $150+028.700+05$

3. $500+026.900+05$

3. $900+025.100+05$

ref $=88 \mathrm{NaOI}$

$2.016+047.700+00$

ref $=84 \mathrm{NaOl}$

ref $=91 \mathrm{El} 01$

3. $560+051.995+00$

$1.274+061.137+00$

ref $=895 a 01$

ref $=81 \mathrm{Wu} 02$

$2.000+011.160+07$

$2.138+011.160+07$

2. $296+011.260+07$

$2.480+011.100+07$

$2,695+01 \quad 1.100+07$

$2.952+011.055+07$

$3.263+019.400+06$

3. $647+017.650+06$

$4.133+015.700+06$

$4.769+014.900+06$

$5.636+013.850+06$

$6.888+012.675+06$

\section{ref $=88 \mathrm{An} 01$}

$1.362+012.450+06$

$1.385+012.750+06$

$1.432+01 \quad 3.100+06$

$1.467+013.250+06$

$1.503+013.300+06$

$1.631+014.060+06$

$1.697+018.400+06$

$1.720+018.910+06$

$1.732+01 \quad 9.240+06$

$1.746+019.150+06$

$1.756+019.600+06$

$1.791+019.510+06$

$1.815+019.650+06$

$1.879+01 \quad 1.260+07$

$2.000+01 \quad 1.340+07$

$2.138+011.300+07$

2. $296+011.230+07$

$2.480+011.190+07$

3. $024+011.100+07$

$3.351+019.900+06$

$3.757+018.600+06$

$4.133+017.550+06$

$4.768+016.150+06$ 
$4.874+016.000+06$

$8.000+012.600+06$

1. $200+021.170+06$

$1.600+026.100+05$

$2.000+023.300+05$

2. $400+02 \quad 2.000+05$

$2.800+021.600+05$

$\mathrm{Z}=8, \mathrm{O}\left(\mathrm{in} \mathrm{O}_{2}\right)$

1. $667+019.030+06$

$\mathrm{Z}=8, \mathrm{O}\left(\mathrm{in} \mathrm{O}_{2}\right)$

1.907+01 1.240+07

$2.033+01 \quad 1.305+07$

2. $175+011.265+07$

$2.339+01 \quad 1.200+07$

$2.530+011.075+07$

$2.755+011.000+07$

$3.024+019.650+06$

$3.351+019.200+06$

3. $757+018.550+06$

$4.275+017.600+06$

$4.959+016.150+06$

$5.904+014.600+06$

$\mathrm{Z}=8,0$

$5.400+045.340+00$

$5.110+052.311+00$

$1.333+061.541+00$

$\mathrm{Z}=11, \mathrm{Na}$ (vapor)

$1.071+033.000+05$

$1.075+032.500+06$

$1.079+036.700+05$

$1.083+037.000+05$

$1.087+037.100+05$

$1.091+037.450+05$

$\mathrm{Z}=12, \mathrm{Mg}$

$2.534+013.141+05$

$2.556+013.355+05$

$2.579+013.706+05$

$2.602+013.147+05$

$2.625+013.218+05$

$2.649+013.205+05$

$2.673+013.356+05$

$2.698+013.258+05$

$2.724+013.333+05$

$2.749+013.450+05$

$2.775+013.266+05$

$2.802+013.394+05$

$2.830+013.537+05$

$2.857+013.418+05$

$2.886+013.368+05$

$2.915+013.419+05$

2. $944+013.421+05$

$2.974+013.364+05$

3. $005+013.289+05$

$3.036+013.334+05$

3. $068+013.290+05$

3. $101+013.252+05$

3. $135+013.310+05$

$5.000+015.770+06$

$9.000+012.060+06$

$1.300+021.000+06$

$1.700+025.200+05$

$2.100+022.800+05$

$2.500+021.800+05$

3 pts

$\begin{array}{lllll}1.685+01 & 1.792+07 & 2.122+01 & 1.148+07\end{array}$

$\mathrm{eV}, \mathrm{b} /$ atom pairs

$1.937+011.235+07$

$2.066+011.340+07$

$2.214+011.290+07$

$2.384+011.195+07$

$2.583+011.005+07$

$2.818+019.850+06$

3. $100+019.550+06$

3. $444+019.050+06$

3. $875+018.350+06$

$4.428+017.250+06$

$5.166+015.750+06$

$6.199+014.200+06$

$\mathrm{eV}, \mathrm{b} / \mathrm{atom}$ pairs

$8.100+04 \quad 4.437+00$

$1.230+053.905+00$

$6.620+052.046+00$

1. $173+061.591+00$

$\mathrm{eV}, \mathrm{b} /$ atom pairs
$1.072+03 \quad 3.000+05$

$1.968+011.290+07$

2. $101+011.030+07$

$2.254+011.270+07$

$2.431+011.145+07$

$2.638+011.030+07$

$2.883+019.800+06$

$3.179+019.450+06$

$3.542+018.900+06$

$4.000+018.150+06$

$4.592+016.900+06$

$5.391+015.350+06$

$6.526+013.900+06$

21 pts

$1.076+03 \quad 4.500+05$

$1.080+037.100+05$

$1.084+037.000+05$

$1088+037.100+05$

$.073+033.200+05$

$1.077+03 \quad 3.800+05$

$1.081+038.000+05$

$1.085+037.900+05$

$1.089+037.400+05$

$\mathrm{eV}, \mathrm{b} /$ atom pairs

226 pts

$2.539+013.211+05$

$2.562+013.092+05$

$2.584+013.222+05$

$2.607+013.179+05$

$2.631+013.206+05$

$2.655+013.421+05$

$2.680+013.230+05$

2. $704+013.425+05$

$2.730+012.412+05$

$2.756+013.330+05$

$2.782+013.397+05$

$2.809+013.361+05$

$2.836+013.263+05$

$2.864+013.275+05$

$2.893+013.479+05$

$2.922+013.326+05$

$2.952+013.284+05$

$2.982+013.296+05$

3. $013+013.398+05$

3. $044+01 \quad 3.247+05$

$3.077+013.239+05$

3. $109+013.394+05$

3. $143+013.275+05$

$2.545+013.204+05$

$2.567+013.288+05$

$2.509+013.189+05$

$2.613+013.268+05$

$2.637+013.114+05$

$2.661+013.390+05$

$2.685+013.340+05$

$2.711+013.404+05$

$2.736+013.536+05$

$2.762+013.649+05$

$2.789+013.208+05$

$2.816+013.428+05$

$2.843+013.434+05$

$2.871+013.350+05$

$2.900+013.383+05$

$2.929+013.240+05$

$2.959+013.378+05$

2. $990+013.321+05$

3. $021+013.380+05$

$3.085+013.295+05$

3. $118+013.361+05$

3. $152+013.242+05$
$6.000+014.440+06$

1. $000+021.650+06$

1. $800+024.500+05$

2. $200+022.500+05$

$2.600+021.700+05$
1. $400+028.400+05$

$7.000+013.430+06$

1. $100+021.390+06$

$1.500+027.300+05$

$1.900+023.800+05$

$2.300+022.200+05$

$2.700+021.700+05$

ref $=895 a 01$

ref $=81 \mathrm{Wu} 02$

$2.000+011.285+07$

$2.138+011.210+07$

$2.296+01 \quad 1.280+07$

$2.480+01 \quad 1.105+07$

$2.695+011.010+07$

$2.952+019.700+06$

3. $263+019.300+06$

$3.647+018.750+06$

$4.133+017.900+06$

$4.769+016.550+06$

$5.636+014.950+06$

$6.888+013.500+06$

ref $=91 \mathrm{E} 101$

$3.560+052.657+00$

1. $274+061.541+00$

ref $=82 \mathrm{Tu} 01$

$1.074+034.900+05$

$1.078+039.000+05$

$1.082+036.900+05$

$1.086+037.000+05$

$1.090+037.400+05$

ref $=94 \mathrm{Gu01}$

$3.052+013.383+05$
$2 \ldots+013.193+05$

$2 \quad+013.183+05$

2... $5+013.071+05$

$2.619+013.418+05$

$2.643+013.333+05$

$2.667+013.249+05$

$2.692+013.575+05$

$2.717+013.228+05$

$2.743+013.469+05$

$2.769+013.301+05$

$2.796+013.413+05$

$2.823+013.472+05$

$2.850+013.555+05$

$2.879+013.423+05$

$2.907+013.814+05$

$2.937+013.306+05$

$2.967+013.239+05$

$2.997+013.315+05$

3. $028+013.458+05$

3. $060+013.312+05$

$3.093+013.320+05$

$3.126+013.295+05$

3. $160+013.310+05$ 
3. $169+013.309+05$ 3. $204+013.143+05$ 3. $239+013.274+05$ $3.276+013.187+05$ $3.313+013.160+05$ 3. $351+013.136+05$ $3.390+013.153+05$ 3. $430+013.046+05$ $3.471+013.099+05$ $3.513+013.057+05$ 3. $556+013.012+05$ 3. $600+012.958+05$ 3. $646+012.902+05$ $3.692+012.915+05$ $3.739+012.760+05$ 3. $788+012.816+05$ $3.838+012.673+05$ $3.889+012.679+05$ 3. $942+012.686+05$ 3. $996+012.587+05$ $4.052+012.505+05$ $4.109+012.364+05$ $4.168+012.332+05$ $4.229+012.253+05$ $4.291+012.145+05$ $4.356+012.103+05$ $4.422+012.022+05$ $4.490+011.943+05$ $4.561+011.891+05$ $4.633+011.840+05$ $4.708+011.795+05$ $4.786+011.790+05$ $4.866+011.796+05$ $4.949+012.219+05$

$\mathrm{Z}=12, \mathrm{Mg}$

$5.893+03 \quad 3.980+03$

$\mathrm{Z}=12, \mathrm{Mg}$

$7.280+061.023+00$

$\mathrm{Z}=13, \mathrm{Al}$

$2.528+013.971+05$ $2.550+013.432+05$ $2.573+013.307+05$

$2.596+014.445+05$

$2.619+014.145+05$

$2.643+014.207+05$

$2.667+013.974+05$

$2.692+013.944+05$

$2.717+014.643+05$

$2.743+01 \quad 4.334+05$

$2.769+01 \quad 3.945+05$

$2.796+013.896+05$

$2.823+014.287+05$

$2.850+014.186+05$

$2.879+014.098+05$

$2.907+014.606+05$

$2.937+014.299+05$

$2.967+014.148+05$

$2.997+013.915+05$

3. $018+014.277+05$
$3.177+013.303+05$ $3.213+013.275+05$ 3. $248+013.212+05$ 3. $285+013.219+05$ 3. $323+013.124+05$ 3. $361+013.191+05$ 3. $400+013.063+05$ $3.441+013.155+05$ $3.482+013.077+05$ 3. $524+012.993+05$ 3. $567+012.927+05$ 3. $612+013.013+05$ 3. $657+012.916+05$ $3.704+012.899+05$ $3.751+012.803+05$ 3. $800+012.754+05$ $3.851+012.709+05$ $3.902+012.704+05$ 3. $956+012.673+05$ $4.010+012.542+05$ $4.066+012.491+05$ 4. $124+012.389+05$ $4.183+012.292+05$ 4. $244+012.216+05$ $4.307+012.152+05$ $4.372+012.039+05$ $4.439+012.003+05$ $4.507+011.931+05$ $4.578+011.879+05$ $4.652+011.829+05$ $4.727+011.781+05$ $4.806+011.772+05$ $4.886+011.829+05$ $4.970+012.457+05$

3. $186+013.259+05$ 3. $221+013.325+05$ $3.258+013.307+05$ $3.294+013.171+05$ $3.332+013.172+05$ $3.371+013.138+05$ 3. $410+013.117+05$ 3. $451+013.101+05$ $3.492+013.104+05$ $3.535+013.009+05$ $3.578+012.994+05$ 3. $623+012.976+05$ $3.669+012.798+05$ $3.715+012.847+05$ 3. $764+012.812+05$ $3.813+012.767+05$ $3.864+012.639+05$ $3.916+012.631+05$ $3.969+012.653+05$ $4.024+012.541+05$ $4.080+012.447+05$ $4.139+012.365+05$ 4. $198+012.303+05$ $4.260+012.188+05$ $4.323+012.153+05$ $4.388+012.043+05$ $4.456+012.001+05$ $4.525+011.956+05$ $4.597+011.908+05$ $4.670+011.797+05$ $4.747+011.797+05$ $4.825+011.782+05$ $4.907+011.880+05$

eV, b/atom pairs

$\begin{array}{llll}1.337+04 & 3.270+02 & 1.744+04 & 1.650+02\end{array}$

$$
\text { ev, b/atom pairs }
$$

1 pt

$\mathrm{eV}, \mathrm{b} / \mathrm{atom}$ pairs

$2.534+013.911+05$

$2.556+013.884+05$

$2.579+013.992+05$

$2.602+014.062+05$

$2.625+013.626+05$

$2.649+013.828+05$

$2.673+013.963+05$

$2.698+014.138+05$

$2.724+014.263+05$

$2.749+01 \quad 4.324+05$

$2.775+01 \quad 4.403+05$

$2.802+01 \quad 4.435+05$

$2.830+014.635+05$

$2.857+01 \quad 4.299+05$

$2.886+014.399+05$

2. $915+014.310+05$

2. $944+014.362+05$

2. $974+014.482+05$

3. $005+014.156+05$

3. $036+014.117+05$
$2.539+014.457+05$

$2.562+013.744+05$

$2.584+013.854+05$

$2.607+014.056+05$

$2.631+013.645+05$

$2.655+013.692+05$

$2.680+013.870+05$

$2.704+014.257+05$

$2.730+013.683+05$

$2.756+014.187+05$

$2.782+014.268+05$

$2.809+014.028+05$

$2.836+014.526+05$

$2.864+014.288+05$

$2.893+014.398+05$

$2.922+014.331+05$

$2.952+014.198+05$

$2.982+014.321+05$

3. $013+014.240+05$

3. $044+014.073+05$
$3.195+013.300+05$

$3.230+013.308+05$

$3.267+013.206+05$

$3.304+013.166+05$

$3.342+013.079+05$

$3.381+013.129+05$

3. $420+013.112+05$

$3.461+013.121+05$

$3.503+013.076+05$

3. $546+012.982+05$

$3.589+012.988+05$

$3.634+012.967+05$

3. $680+012.878+05$

$3.727+012.883+05$

$3.776+012.738+05$

$3.825+012.734+05$

$3.876+012.724+05$

3. $929+012.663+05$

$3.983+012.564+05$

$4.038+012.491+05$

$4.094+012.444+05$

$4.153+012.293+05$

$4.214+012.267+05$

$4.275+012.186+05$

$4.339+012.111+05$

$4.405+012.038+05$

$4.473+011.999+05$

$4.543+011.923+05$

$4.615+011.852+05$

$4.689+011.778+05$

$4.766+011.791+05$

$4.846+011.790+05$

$4.928+012.017+05$

ref $=88 \mathrm{NaO} 01$

$2.016+048.900+01$

ref $=84 \mathrm{YeOI}$

ref $=94 \mathrm{GuO} 1$

$2.545+01 \quad 3.284+05$

$2.567+014.122+05$

$2.590+013.907+05$

2. $613+014.300+05$

$2.637+013.879+05$

$2.661+014.233+05$

$2.686+013.696+05$

$2.711+014.066+05$

$2.736+014.294+05$

$2.762+014.187+05$

$2.789+014.208+05$

$2.816+014.162+05$

$2.843+014.239+05$

$2.871+014.243+05$

$2.900+014.258+05$

$2.929+014.105+05$

$2.959+014.037+05$

$2.990+014.051+05$

3. $021+014.500+05$

3. $052+014.318+05$ 
$3.060+014.004+05$

3.093 ग $4.389+05$

3. 126 ग $4.397+05$

3. $160+14.117+05$

3. $195+014.114+05$

3. $230+014.263+05$

3. $267+014.175+05$

3. $304+01 \quad 4.203+05$

3. $342+013.994+05$

$3.381+014.124+05$

3. $420+014.110+05$

3. $461+013.981+05$

$3.503+014.153+05$

$3.546+013.973+05$

3. $589+014.061+05$

3. $634+014.008+05$

3. $680+014.111+05$

3. $727+013.962+05$

3. $776+014.020+05$

3. $825+014.119+05$

$3.876+01 \quad 3.985+05$

$3.929+014.013+05$

3. $983+013.884+05$

$4.038+014.109+05$

$4.095+013.853+05$

$4.153+013.880+05$

$4.213+013.716+05$

$4.275+013.810+05$

$4.339+013.750+05$

$4.405+013.758+05$

$4.473+013.704+05$

$4.543+013.641+05$

$4.615+013.660+05$

$4.689+013.508+05$

$4.766+013.516+05$

$4.846+013.302+05$

$4.928+013.185+05$

$5.013+013.000+05$

$5.101+012.966+05$

$5.192+012.952+05$

$5.286+012.825+05$

$5.384+012.684+05$

$5.486+012.770+05$

$5.591+012.616+05$

$5.701+012.487+05$

$5.815+012.431+05$

$5.933+012.383+05$

$6.057+012.312+05$

$6.186+012.213+05$

$6.320+012.214+05$

$6.461+012.333+05$

$6.608+012.316+05$

$6.761+012.418+05$

$6.922+012.525+05$

$7.091+012.570+05$

$7.268+013.249+05$

$Z=13, A 1$

2. $045+021.838+06$

2. $084+021.872+06$

2. $124+021.876+06$

2. $165+021.800+06$
3. $068+014.285+05$

3. $101+014.461+05$

3. $135+014.445+05$

3. $169+014.254+05$

3. $204+014.058+05$

$3.239+014.084+05$

3. $276+01 \quad 4.190+05$

3. $313+014.102+05$

3. $351+014.138+05$

3. $390+014.089+05$

3. $430+014.032+05$

3. $471+01 \quad 4.078+05$

3. $513+014.222+05$

3. $556+013.942+05$

3. $600+014.151+05$

3. $646+01 \quad 4.403+05$

3. $692+014.252+05$

$3.739+014.292+05$

$3.788+014.111+05$

3. $838+014.064+05$

$3.889+013.752+05$

3. $942+014.006+05$

$3.996+013.969+05$

$4.052+013.961+05$

$4.109+013.841+05$

$4.168+013.905+05$

$4.229+013.769+05$

$4.291+013.811+05$

$4.356+013.784+05$

$4.422+013.819+05$

$4.490+013.690+05$

$4.560+013.729+05$

$4.633+013.575+05$

$4.708+013.553+05$

$4.786+013.365+05$

$4.866+013.194+05$

$4.949+013.087+05$

$5.034+013.028+05$

$5.123+012.921+05$

$5.215+012.882+05$

$5.310+012.811+05$

$5.409+012.739+05$

$5.512+012.709+05$

$5.618+012.582+05$

$5.729+012.578+05$

$5.844+012.426+05$

$5.964+012.391+05$

$6.089+012.179+05$

$6.219+012.180+05$

$6.354+012.238+05$

$6.497+012.290+05$

$6.645+012.294+05$

$6.801+012.509+05$

$6.964+012.537+05$

$7.135+012.512+05$
$3.077+014.080+05$

3. $109+014.173+05$

3. $143+01 \quad 4.264+05$

3. $177+014.358+05$

$3.213+014.039+05$

3. $248+01 \quad 4.285+05$

3. $285+014.206+05$

3. $323+014.113+05$

3. $361+014.159+05$

3. $400+014.099+05$

$3.441+012.966+05$

$3.482+014.231+05$

3. $524+013.994+05$

3. $567+013.827+05$

3. $612+013.968+05$

3. $657+01 \quad 4.245+05$

3. $704+014.220+05$

$3.751+014.095+05$

3. $800+014.146+05$

$3.851+014.066+05$

$3.902+01 \quad 4.082+05$

$3.956+01 \quad 4.040+05$

4. $010+014.117+05$

$4.066+013.985+05$

$4.124+013.907+05$

$4.183+013.893+05$

$4.244+013.790+05$

$4.307+013.872+05$

$4.372+013.807+05$

$4.439+013.837+05$

$4.507+013.709+05$

$4.578+013.745+05$

$4.652+013.593+05$

$4.727+013.591+05$

$4.806+013.318+05$

$4.886+013.216+05$

$4.970+013.105+05$

$5.056+012.999+05$

5. $146+013.002+05$

$5.238+012.794+05$

$5.335+012.825+05$

$5.434+012.702+05$

$5.538+012.566+05$

$5.645+012.568+05$

$5.757+012.500+05$

$5.873+012.410+05$

$5.995+012.255+05$

$6.121+012,267+05$

$6.252+012.199+05$

$6.390+012.164+05$

$6.533+012.242+05$

$6.684+012.331+05$

$6.841+012.492+05$

$7.006+012.498+05$

$7.179+012.634+05$
$3.085+014.296+05$

3. $118+014.057+05$

3. $152+014.132+05$

3. $186+014.158+05$

3. $221+014.042+05$

3. $258+014.201+05$

3. $294+014.020+05$

3. $332+014.040+05$

3. $371+01 \quad 4.411+05$

3. $410+01 \quad 4.089+05$

3. $451+013.906+05$

$3.492+013.965+05$

$3.535+013.919+05$

$3.578+013.914+05$

$3.623+014.202+05$

$3.669+014.329+05$

3. $715+014.282+05$

$3.764+013.980+05$

3. $813+014.108+05$

3. $864+014.079+05$

$3.916+014.028+05$

$3.969+013.995+05$

$4.024+013.85 \% 05$

$4.080+013.9 ;-05$

$4.139+013.89 \div+05$

$4.198+013.771+05$

$4.260+013.733+05$

$4.323+013.703+05$

$4.388+013.772+05$

$4.456+013.750+05$

$4.525+013.719+05$

$4.597+013.612+05$

$4.670+013.607+05$

$4.747+013.484+05$

$4.825+013.287+05$

$4.907+013.227+05$

$4.991+013.061+05$

$5.078+012.955+05$

$5.169+012.812+05$

$5.262+012.822+05$

$5.359+012.794+05$

$5.460+012.702+05$

$5.564+012.609+05$

$5.673+012.559+05$

$5.786+012.461+05$

$5.903+012.365+05$

$6.026+012.323+05$

$6.153+012.138+05$

$6.286+012.218+05$

$6.425+012.214+05$

$6.570+012.375+05$

$6.722+012.338+05$

$6.881+012.449+05$

$7.048+012.515+05$

$7.223+012.588+05$

ref $=90 \mathrm{Fu01}$

$2.074+021.876+06$

2. $114+021.879+06$

2. $155+021.822+06$

$2.197+021.718+06$ 
$2.208+021.690+06$

$2.251+021.615+06$

2. $297+021.564+06$

$2.343+021.505+06$

$2.391+021.460+06$

$2.441+021.410+06$

$2.492+021.350+06$

$2.544+021.308+06$

$2.599+021.270+06$

$2.655+021.217+06$

$2.714+021.132+06$

$2.774+021.096+06$

$2.836+021.027+06$

$2.901+024.036+05$

$2.967+023.765+05$

3. $037+025.082+05$

3. $108+026.857+05$

$3.183+025.677+05$

3. $260+026.326+05$

$3.340+036.369+05$

3. $424+026.191+05$

$3.510+025.912+05$

3. $600+025.700+05$

3. $694+025.297+05$

3. $791+024.959+05$

3. $893+024.594+05$

$3.999+024.507+05$

4.110+02 2. $451+05$

4. $196+028.849+04$

$4.286+029.145+04$

$4.380+029.396+04$

$4.476+029.167+04$

$4.577+021.081+05$

4. $681+023.325+05$

$4.788+02 \quad 2.687+05$

$4.900+022.652+05$

$5.015+02 \quad 2.547+05$

$5.135+02 \quad 2.500+05$

$5.260+022.312+05$

$5.390+02 \quad 2.108+05$

$5.524+022.238+05$

$5.664+022.124+05$

$5.810+021.985+05$

$5.962+021.976+05$

$6.121+021.711+05$

$6.286+021.538+05$

$6.458+021.458+05$

$6.638+021.397+05$

$6.827+021.280+05$

7. $024+021.182+05$

$7.230+021.068+05$

$7.446+029.638+04$

$7.673+029.275+04$

$7.912+028.625+04$

$8.163+027.532+04$

$8.426+027.075+04$

$8.704+026.362+04$

$8.998+02 \quad 5.435+04$

9. $307+024.485+04$

$9.635+025.686+04$
$2.219+021.670+06$

$2.263+021.602+06$

$2.308+021.552+06$

$2.355+021.486+06$

$2.403+021.453+06$

$2.453+021.396+06$

$2.505+021.336+06$

$2.558+021.296+06$

$2.613+021.256+06$

$2.670+021.199+06$

$2.728+021.129+06$

$2.789+021.070+06$

$2.852+028.776+05$

$2.917+023.946+05$

$2.985+024.070+05$

3. $054+025.912+05$

3. $127+026.875+05$

3. $202+026.602+05$

3. $280+026.365+05$

3. $361+026.342+05$

$3.445+026.126+05$

$3.532+025.834+05$

$3.623+025.658+05$

3. $718+025.228+05$

$3.816+02 \quad 4.874+05$

3. $919+024.500+05$

$4.026+023.599+05$

$4.138+022.731+05$

$4.218+02 \quad 8.939+04$

$4.309+029.342+04$

4. $404+029.310+04$

$4.501+029.512+04$

$4.602+021.109+05$

$4.707+02 \quad 2.329+05$

$4.816+02 \quad 2.662+05$

4. $928+022.631+05$

$5.045+022.565+05$

5. $166+02 \quad 2.443+05$

$5.292+022.298+05$

$5.423+022.489+05$

$5.559+022.233+05$

$5.700+022.099+05$

$5.848+022.131+05$

$6.001+021.989+05$

$6.161+021.679+05$

6. $328+021.474+05$

$6.503+021.472+05$

$6.684+021.352+05$

$6.875+021.255+05$

$7.075+021.159+05$

$7.283+021.047+05$

$7.502+029.333+04$

$7.732+029.293+04$

$7.973+028.253+04$

$8.227+027.326+04$

$8.494+026.972+04$

$8.776+025.856+04$

$9.073+026.389+04$

$9.387+026.116+04$

$9.719+02 \quad 5.628+04$
$2.229+021.646+06$

2. $274+021.591+06$

2. $320+021.535+06$

$2.367+021.478+06$

$2.416+021.446+06$

$2.466+021.382+06$

$2.518+021.325+06$

$2.571+021.288+06$

$2.627+021.240+06$

$2.684+021.170+06$

2. $743+021.129+06$

$2.805+021.054+06$

$2.868+026.688+05$

$2.934+02 \quad 3.800+05$

3. $002+024.218+05$

$3.072+026.485+05$

3. $145+026.770+05$

3. $221+026.546+05$

$3.300+026.354+05$

$3.381+026.296+05$

$3.466+026.063+05$

$3.555+025.809+05$

3. $647+025.514+05$

3. $742+025.143+05$

$3.842+024.792+05$

3. $945+024.356+05$

$4.054+023.186+05$

$4.167+021.888+05$

$4.240+028.791+04$

$4.332+029.234+04$

$4.428+029.337+04$

$4.526+029.620+04$

$4.628+022.114+05$

$4.734+022.533+05$

$4.843+022.653+05$

$4.957+022.628+05$

$5.075+022.533+05$

$5.197+02 \quad 2.411+05$

$5.324+021.492+05$

$5.456+022.651+05$

$5.594+022.208+05$

$5.737+02 \quad 2.057+05$

$5.885+02 \quad 2.024+05$

$6.041+021.914+05$

$6.202+021.648+05$

$6.371+021.451+05$

$6.547+021.488+05$

$6.731+021.324+05$

$6.924+021.225+05$

7. $126+021.148+05$

$7.337+02 \quad 1.028+05$

$7.559+029.378+04$

$7.791+029.024+04$

$8.035+028.056+04$

$8.292+027.227+04$

$8.563+026.671+04$

$8.849+025.542+04$

9. $150+020.000+00$

$9.468+021.538+05$

9. $805+025.744+04$
2. $240+021.623+06$

2. $285+021.583+06$

2. $331+021.518+06$

$2.379+021.471+06$

$2.428+021.425+06$

$2.479+021.361+06$

2. $531+021.320+06$

$2.585+021.280+06$

$2.641+021.223+06$

2. $699+021.148+06$

$2.759+021.115+06$

$2.820+029.862+05$

$2.884+024.694+05$

$2.950+023.659+05$

3. $019+024.453+05$

3. $090+026.764+05$

3. $164+026.726+05$

3. $241+026.447+05$

$3.320+026.378+05$

3. $402+026.262+05$

$3.488+026.108+05$

$3.577+025.758+05$

$3.670+025.408+05$

3. $767+025.053+05$

$3.867+024.698+05$

$3.972+024.191+05$

$4.082+022.864+05$

$4.196+021.855+05$

$4.263+029.033+04$

$4.356+029.328+04$

$4.452+029.369+04$

$4.551+029.938+04$

$4.654+022.583+05$

$4.761+02 \quad 2.682+05$

$4.871+022.657+05$

$4.986+022.589+05$

$5.105+022.529+05$

$5.228+02 \quad 2.480+05$

$5.357+021.788+05$

$5.490+022.248+05$

$5.629+022.185+05$

$5.773+022.040+05$

$5.923+02$ I. $996+05$

$6.080+021.832+05$

$6.244+021.642+05$

$6.414+021.556+05$

$6.593+021.483+05$

$6.779+021.314+05$

$6.974+021.203+05$

$7.177+021.076+05$

$7.391+029.965+04$

$7.616+029.324+04$

$7.851+028.379+04$

$8.099+027.527+04$

$8.359+027.052+04$

$8.633+026.152+04$

$8.923+025.192+04$

9. $228+025.466+04$

$9.551+025.153+03$

$9.892+025.677+04$ 
$Z=13, A 1$

$5.893+035.750+03$

$Z=13, A 1$

$5.895+03 \quad 5.445+03$

$\mathrm{Z}=13, \mathrm{Al}$

$5.947+035.269+03$

$\mathrm{Z}=13, \mathrm{Al}$

$6.400+034.310+03$

$Z=13, \mathrm{Al}$

$6.460+03 \quad 4.265+03$

$\mathrm{Z}=13, \mathrm{Al}$

$8.118+032.217+03$

$Z=13, A I$

1. $343+045.243+02$

5. $920+041.138+01$

$\mathrm{Z}=13, \mathrm{Al}$

2. $260+041 \cdot 120+02$

$Z=13, A 1$

3. $080+04 \quad 4.700+01$

3. $030+054.460+00$

$Z=13, A 1$

$4.300+042.168+01$

$9.900+047.844+00$

$\mathrm{Z}=13, \mathrm{Al}$

5. $240+041.400+01$

$\mathrm{Z}=13, \mathrm{Al}$

7. $280+061.117+00$

$\mathrm{Z}=14, \mathrm{Si}$

4. $959+016.539+05$

$5.040+016.318+05$

$5.123+016.318+05$

$5.209+016.229+05$

$5.298+015.997+05$

$5.390+016.164+05$

$5.486+015.893+05$

$5.585+015.793+05$

$5.687+015.846+05$

$5.793+015.744+05$

$5.904+015.869+05$

$6.018+015.867+05$

$6.138+015.938+05$

$6.262+015.948+05$

$6.391+015.958+05$

$6.525+015.948+05$

$6.666+015.954+05$

$6.812+015.886+05$

$6.965+015.762+05$

$7.125+015.592+05$

$7.293+015.136+C 5$

$7.469+014.823+05$
$\mathrm{eV}, \mathrm{b} / \mathrm{atom}$ pairs

$1.337+04 \quad 4.600+02 \quad 1.744+04 \quad 2.250+02$

$\mathrm{eV}, \mathrm{b} / \mathrm{atom}$ pairs

$8.041+032.261+03$

eV, b/atom pairs

$6.460+034.229+03$

eV, b/atom pairs

1 pts

eV, b/atom pairs

1 pts

eV, b/atom pairs

1 pts

5 pts

eV, b/atom pairs

1. $722+04 \quad 2.576+02 \quad 2.016+04$

$\mathrm{eV}, \mathrm{b} /$ atom pairs

1 pts

7 pts

$\mathrm{eV}, \mathrm{b} / \mathrm{atom}$ pairs

$3.500+04 \quad 3.600+01 \quad 8.100+04 \quad 8.650+00$

$3.560+05 \quad 4.230+00 \quad 3.840+05$

$\mathrm{eV}, \mathrm{b} /$ atom pairs

$4.100+00$

$5.300+04 \quad 1.572+01 \quad 5.950+04 \quad 1.245+01$ $1.220+056.690+00 \quad 1.520+05$

$\mathrm{eV}, \mathrm{b} /$ atom pairs

1 pts

eV, b/atom pairs

$1 \mathrm{pt}$

$\mathrm{eV}, \mathrm{b} /$ atom pairs

126 pts

$4.979+016.223+05 \quad 4.999+016.409+05$

$5.060+016.217+05$

$5.081+016.222+05$

$5.144+016.352+05$

$5.231+016.156+05$

$5.321+016.104+05$

$5.414+015.927+05$

$5.510+015.908+05$

$5.610+015.940+05$

$5.713+015.790+05$

$5.821+015.757+05$

$5.932+015.805+05$

$6.048+015.944+05$

$6.168+016.006+05$

$6.293+015.983+05$

$6.424+015.985+05$

$6.560+015.926+05$

$6.701+015.901+05$

$6.850+015.844+05$

$7.004+015.776+05$

$7.166+015.557+05$

$7.336+015.131+05$

$7.514+01 \quad 4.714+05$
$5.166+016.148+05$

$5.253+016.285+05$

5. $344+016.120+05$

$5.438+015.954+05$

$5.535+015.837+05$

$5.635+015.871+05$

$5.740+015.823+05$

$5.848+015.835+05$

$5.961+015.862+05$

$6.077+015.875+05$

$6.199+016.015+05$

$6.326+015.965+05$

$6.457+015.994+05$

$6.595+015.991+05$

$6.738+015.903+05$

$6.888+015.813+05$

$7.044+015.681+05$

$7.208+015.438+05$

$7.380+015.009+05$

$7.560+015.576+05$ ref $=88 \mathrm{NaOI}$

$2.016+041.200+02$

ref $=94 \mathrm{KeOI}$

ref $=92 \mathrm{KeO} 2$

ref $=93 \mathrm{KeO} 2$

ref $=93 \mathrm{KeOI}$

ref $=93 \mathrm{KeO} 3$

ref $=84 \mathrm{NaOI}$

$4.652+041.756+01$

ref $=90$ Qu01

ref $=93 \mathrm{NaO} 01$

2. $760+054.580+00$

ref $=87 \mathrm{MaOI}$

$8.100+048.975+00$

ref $=89 \mathrm{Ke} 01$

ref $=84 \mathrm{YeOI}$

ref $=94 \mathrm{GuO1}$

$5.019+016.092+05$

$5.102+016.492+05$

$5.187+016.320+05$

$5.276+016.285+05$

$5.367+016.039+05$

$5.462+016.014+05$

$5.560+015.799+05$

$5.661+015.815+05$

$5.767+015.809+05$

$5.876+01 \quad 5.832+05$

$5.989+015.840+05$

$6.107+015.878+05$

$6.230+015.959+05$

$6.358+015.968+05$

$6.491+016.008+05$

$6.630+015.964+05$

$6.775+015.903+05$

$6.926+015.768+05$

$7.085+015.638+05$

$7.250+015.353+05$

$7.424+01 \quad 4.961+05$

$7.606+014.509+05$ 
$7.653+01 \quad 4.413+05$ $7.847+013.976+05$ $8.051+013.677+05$ $8.265+013.527+05$ $8.492+013.459+05$ $8.731+013.433+05$ $8.984+013.402+05$ 9.252+01 $3.427+05$ $9.537+013.493+01$ 9. $840+013.555+05$

$\mathrm{Z}=14, \mathrm{Si}$

$1.486+03 \quad 2.746+04$

3. $313+03 \quad 3.378+04$ $4.950+031.124+04$ $7.472+033.681+03$ $8.905+032.185+03$ 1. $054+041.336+03$ $1.214+048.695+02$ $1.338+046.444+02$ 1. $517+04 \quad 4.431+02$ 1. $744+042.991+02$ $1.997+042.041+02$ $2.519+041.030+02$ $2.847+047.455+01$

$\mathrm{Z}=14, \mathrm{Si}$

$5.893+037.280+03$

$Z=14, \mathrm{Si}$

$4.652+042.155+01$

$\mathrm{Z}=18, \mathrm{Ar}$

$\begin{array}{ll}1.667+01 & 3.231+07\end{array}$

$\mathrm{Z}=18, \mathrm{Ar}$

3. $263+022.578+06$ 4. $133+021.809+06$ $5.391+021.024+06$ $6.512+026.680+05$ $8.244+023.650+05$

$\mathrm{Z}=22, \mathrm{Ti}$

$4.000+023.180+05$ $4.530+022.703+06$ $4.800+021.407+06$ $5.500+021.431+06$ $7.500+028.746+05$

\section{$Z=22, \mathrm{Ti}$}

$4.000+02 \quad 4.200+05$ $4.200+023.700+05$ $4.400+023.600+05$ $4.540+024.750+05$ $4.610+021.780+06$ $4.680+022.010+06$ 4. $760+021.720+06$ $4.840+021.490+06$ $4.920+021.440+06$ $5.000+021.475+06$ $5.080+021.500+06$ $5.160+021.440+06$
$7.701+014.255+05$

$7.897+013.881+05$

$8.103+013.627+05$

$8.321+013.498+05$

$8.550+013.442+05$

$8.793+013.418+05$

9. $050+013.417+05$

$9.322+013.442+05$

9. $611+013.505+05$

$9.918+013.622+05$

$\mathrm{eV}, \mathrm{b} /$ atom pairs

$7.749+014.201+05$ $7.947+013.857+05$ $8.157+013.592+05$ $8.377+013.493+05$ 8. $610+013.461+05$ $8.856+013.424+05$ $9.116+013.394+05$ $9.392+013.469+05$ $9.686+013.512+05$ $1.740+031.789+04$ $3.590+032.807+04$ $5.427+038.429+03$ $8.041+032.963+03$ $9.185+031.993+03$ $1.121+041.093+03$ $1.231+048.396+02$ $1.377+045.913+02$ 1. $658+043.443+02$ 1. $862+042.442+02$ 2. $210+041.505+02$ 2. $546+041.025+02$ $2.911+047.269+01$

$\mathrm{eV}, \mathrm{b} /$ atom pairs

$2.622+036.681+04$ $4.509+031.489+04$ $6.399+035.703+03$ $8.265+032.717+03$ $9.707+031.667+03$ 1. $148+041.025+03$ $1.262+047.893+02$ $1.476+04 \quad 4.860+02$ $1.673+043.382+02$ $1.895+042.279+02$ $2.414+041.160+02$ $2.726+048.154+01$

$1.337+04 \quad 6.300+02 \quad 1.744+04 \quad 3.500+02$

$\mathrm{eV}, \mathrm{b} / \mathrm{atom}$ pairs

5. $920+041.301+01$

2 pts

$\mathrm{eV}, \mathrm{b} / \mathrm{atom}$ pairs

$\begin{array}{lllll}1.685+01 & 3.266+07 & 2.122+01 & 3.598+07\end{array}$

$\mathrm{eV}, \mathrm{b} / \mathrm{atom}$ pairs

19 pts

$\begin{array}{llll}3.444+02 & 2.480+06 & 3.647+02 & 2.259+06\end{array}$ $4.428+02 \quad 1.527+06 \quad 4.769+02 \quad 1.305+06$ $5.636+029.330+05 \quad 5.904+02 \quad 8.300+05$ $6.873+02 \quad 5.800+05 \quad 7.276+02 \quad 5.050+05$ $8.831+02 \quad 3.150+05 \quad 9.508+02 \quad 2.620+05$

$\mathrm{eV}, \mathrm{b} / \mathrm{atom}$ pairs

20 pts

$4.450+02 \quad 1.988+05 \quad 4.500+02 \quad 2.862+06$ $4.550+02 \quad 5.168+06 \quad 4.600+02 \quad 2.385+06$ $4.900+021 \cdot 574+06$ $6.000+021.352+06$ $8.000+028.348+05$

$5.000+021.511+06$ $6.500+021.193+06$ $8.500+026.361+05$

$\mathrm{eV}, \mathrm{b} / \mathrm{atom}$ pairs

87 pts

4.040+02 3.900+05 4.100+02 3.800+05 $4.250+02 \quad 3.500+05 \quad 4.300+02 \quad 3.500+05$ $4.450+02 \quad 3.600+05 \quad 4.500+02 \quad 3.700+05$ $4.560+026.500+05 \quad 4.580+02 \quad 1.000+06$ $4.620+02 \quad 1.880+06 \quad 4.640+02 \quad 2.000+06$ $4.700+02 \quad 1.940+06 \quad 4.720+02 \quad 1.830+06$ $4.780+02 \quad 1.610+06 \quad 4.800+02 \quad 1.580+06$ $4.860+02 \quad 1.475+06 \quad 4.880+02 \quad 1.450+06$ $4.940+021.430+06 \quad 4.960+02 \quad 1.450+06$ $5.020+02 \quad 1.490+06 \quad 5.040+02 \quad 1.490+06$ $5.100+021.400+06 \quad 5.120+02 \quad 1.450+06$ $5.180+021.420+06 \quad 5.200+02 \quad 1.400+06$
$7.797+013.297+05$

$7.999+013.705+05$

$8.211+013.522+05$

$8.434+013.470+05$

$8.670+013.451+05$

$8.919+013.420+05$

9. $184+013.414+05$

$9.464+013.462+05$

$9.762+013.535+05$

ref $=95 \mathrm{WaOl}$

$2.816+03 \quad 4.997+04$

4. $932+031.146+04$

$7.058+034.282+03$

$8.494+032.507+03$

$1.031+041.399+03$

1. $192+049.324+02$

$1.302+047.423+02$

$1.493+04 \quad 4.701+02$

$1.702+043.336+02$

1. $960+042.111+02$

$2.493+041.048+02$

$2.786+047.781+01$

ref $=88 \mathrm{NaO1}$

$2.016+041.800+02$

ref $=84 \mathrm{NaOI}$

ref $=89 \mathrm{SaO1}$

ref $=87 \mathrm{Ya01}$

$3.875+021.987+06$

5. $166+021.116+06$

$6.190+027.370+05$

$7.730+024.320+05$

ref $=90$ De01

$4.520+024.413+06$

4. $700+021.431+06$

5. $250+021.471+06$

7. $000+029.939+05$

$9.000+025.566+05$

ref $=87 \mathrm{De} 02$

$4.150+023.800+05$

$4.350+023.500+05$

$4.520+024.000+05$

$4.600+021.500+06$

$4.660+022.020+06$

4. $740+021.790+06$

$4.820+021.540+06$

$4.900+021.450+06$

$4.980+021.450+06$

5. $060+021.490+06$

5. $140+021.450+06$

$5.220+021.390+06$ 
$5.240+021 \cdot 360+06$

$5.320+021 \cdot 360+06$

$5.400+021.410+06$

$5.480+021.400+06$

$5.560+021.380+06$

$5.640+021.375+06$

$5.720+021.365+06$

$5.800+021.400+06$

$5.880+021 \cdot 390+06$

$5.960+021.375+06$

$\mathrm{Z}=22, \mathrm{Ti}$

$4.509+038.865+03$

$5.412+03 \quad 4.866+04$

$6.490+032.902+04$

$7.649+03 \quad 1.860+04$

$8.905+031.239+04$

$\mathrm{Z}=22, \mathrm{~T} i$

$4.966+03 \quad 3.282+04$

$\mathrm{Z}=22, \mathrm{Ti}$

2. $260+049.939+02$

$\mathrm{Z}=23, \quad \mathrm{~V}$

$4.509+031.106+04$

$5.412+03 \quad 6.446+03$

$6.925+032.817+04$

$8.041+031.879+04$

$9.572+031.194+04$

$\mathrm{Z}=24, \mathrm{Cr}$

$2.045+021.958+06$

2. $084+021.928+06$

$2.124+021.906+06$

$2.165+021.880+06$

2. $208+021.845+06$

$2.251+021.785+06$

$2.297+021.729+06$

$2.343+021.677+06$

$2.391+021.620+06$

$2.441+021.572+06$

$2.492+021.516+06$

$2.544+021.458+06$

$2.599+021.403+06$

$2.655+021.336+06$

$2.714+021.263+06$

$2.774+021.230+06$

$2.836+021.172+06$

$2.901+021.391+06$

$2.967+021.354+06$

$3.037+021.204+06$

$3.108+021.106+06$

3. $183+021.062+06$

3. $260+021.025+06$

$3.340+039.605+05$

3. $424+029.475+05$

$3.510+028.984+05$

$3.600+028.365+05$

$3.694+028.014+05$

$3.791+027.689+05$

$3.893+027.474+05$
$5.260+021.350+06$

5. $340+021.380+06$

$5.420+021.410+06$

$5.500+021.400+06$

$5.580+021.380+06$

$5.660+021.370+06$

$5.740+021.370+06$

$5.820+021.400+06$

$5.900+021.380+06$

$5.980+021.390+06$

$\mathrm{eV}, \mathrm{b} / \mathrm{atom}$ pairs

$4.932+03 \quad 6.820+03 \quad 4.950+03 \quad 7.076+03$

$5.895+03 \quad 3.800+04$

$6.925+032.401+04$

$8.041+031.629+04$

$5.945+03 \quad 3.729+04$

$7.058+032.377+04$

$8.265+031.519+04$

$9.572+031.011+04$

$9.876+039.302+03$

$\mathrm{eV}, \mathrm{b} / \mathrm{atom}$ pairs

$4.975+036.130+04 \quad 5.500+034.278+04$

eV, b/atom pairs

1 pts

$\mathrm{eV}, \mathrm{b} / \mathrm{atom}$ pairs

19 pts

$4.932+038.552+03$

$5.895+034.399+04$

$7.058+032.690+04$

$8.265+031.760+04$

$9.876+031.076+04$

$4.950+038.561+03$

$6.400+03 \quad 3.494+04$

$7.476+032.300+04$

$8.631+031.556+04$

$1.098+047.994+03$

eV, b/atom pairs

256 pts

$2.055+02 \quad 1.952+06$

$2.064+021.947+06$

$2.094+021.922 \div 06$

$2.134+021.897+06$

$2.176+021.872+06$

$2.219+021.832+06$

$2.263+021.774+06$

$2.308+021.720+06$

$2.355+021.654+06$

$2.403+021.608+06$

$2.453+021.558+06$

$2.505+021.507+06$

$2.558+021.445+06$

$2.613+021.377+06$

$2.670+021.311+06$

$2.728+021.261+06$

$2.789+021.221+06$

$2.852+021.228+06$

$2.917+021.386+06$

2. $985+021.316+06$

3. $054+021.158+06$

$3.127+021.099+06$

3. $202+021.034+06$

3. $280+021.017+06$

3. $361+029.469+05$

3. $445+029.403+05$

$3.532+028.810+05$

$3.623+028.236+05$

$3.718+027.988+05$

3. $816+027.634+05$

3. $919+027.410+05$
2. $104+021.921+06$

$2.144+021.977+06$

$2.186+021.866+06$

$2.229+021.818+06$

$2.274+021.754+06$

$2.320+021.699+06$

$2.367+021.642+06$

$2.466+021.546+06$

$2.518+021.491+06$

$2.571+021.431+06$

$2.627+021.353+06$

$2.684+021.293+06$

2. $743+021.255+06$

$2.805+021.220+06$

$2.868+02$ 1. $326+06$

$2.934+021.420+06$

3. $002+021.272+06$

$3.072+021.141+06$

$3.145+021.121+06$

3. $221+021.034+06$

3. $300+021.007+06$

$3.381+029.344+05$

3. $466+029.322+05$

$3.555+028.623+05$

3. $647+028.054+05$

$3.742+027.957+05$

3. $945+027.425+05$
$2.416+02 \quad 1.597+06$

3. $842+027.732+05$
$5.300+02 \quad 1.370+06$

$5.380+021.400+06$

$5.460+021.390 \div 06$

$5.540+021.400+06$

$5 \cdot 620+021 \cdot 350 \div 06$

$5.700+021.360+06$

$5.780+021.390+06$

$5.860+021.370+06$

$5.940+021.400+06$

ref $=89$ Uno1

$5.427+03 \quad 4.834+04$

$6.400+033.085+04$

$7.476+031.959+04$

$8.631+031.336+04$

$1.098+046.750+03$

ref $=88 \mathrm{~Pa} 01$

ref $=900 u 01$

ref $=89$ Uno1

$5.427+036.480 \div 03$

$6.490+03 \quad 3.460+04$

$7.649+032.174+04$

$8.905+031.447+04$

ref $=90$ Fuoz

$2.074+02 \quad 1.935+06$

$2.114+021.908+06$

$2.155+021.887+06$

$2.197+021.855+06$

2. $240+021.802+06$

$2.285+021.742+06$

$2.331+021.687+06$

$2.379+021.628+06$

$2.428+021.584+06$

$2.479+021.533+06$

$2.531+021.475+06$

$2.585+02$ I. $420+06$

$2.641+021.325+06$

$2.699+02 \quad 1.281+06$

$2.759+021.243+06$

2. $820+021.417+06$

$2.884+021.437+06$

2. $950+021.403+06$

$3.019+021.235+06$

$3.090+021.116+06$

$3.164+021.064+06$

3. $241+02 \quad 1.043+06$

$3.320+029.880+05$

$3.402+029.488+05$

$3.488+029.120+05$

$3.577+02 \quad 8.463+05$

$3.670+028.055+05$

$3.767+027.858+05$

$3.867+027.584+05$

$3.972+027.534+05$ 
3. $999+027.503+05$

$4.110+028.895+05$

$4.196+021.216+06$

$4.286+021.127+06$

$4.380+021.050+06$

$4.476+029.767+05$

$4.577+029.084+05$

$4.681+026.171+05$

$4.788+02 \quad 5.548+05$

$4.900+025.408+05$

$5.015+024.958+05$

$5.135+024.709+05$

5. $260+024.499+05$

5. $390+024.651+05$

$5.524+024.383+05$

$5.664+02 \quad 3.175+05$

5. $810+022.590+06$

5. $962+022.197+06$

$6.121+021.982+06$

$6.286+021.853+06$

$6.458+021.812+06$

$6.638+021.719+06$

$6.827+021.667+06$

$7.024+021.750+06$

$7.230+021.602+06$

$7.446+021.565+06$

$7.673+021.517+06$

$7.912+021.382+06$

$8.163+021.293+06$

$8.426+021.257+06$

$8.704+021.149+06$

$8.998+021.052+06$

$9.307+028.077+05$

$9.635+029.971+05$

$\mathrm{Z}=24, \mathrm{Cr}$

5. $000+025.100+05$

$5.400+024.800+05$

$5.700+025.600+05$

$5.780+021.150+06$

$5.860+022.250+06$

$5.940+022.250+06$

$6.020+021.820+06$

$6.100+021.680+06$

$6.180+021.630+06$

6. $260+021.620+06$

$6.340+021.620+06$

$6.420+021.600+06$

$6.500+021.550+06$

$6.580+021.520+06$

$6.660+021.540+06$

$6.740+021.520+06$

$6.820+021.480+06$

$6.900+021.460+06$

$6.980+021.460+06$

$Z=24, \mathrm{Cr}$

$5.000+02 \quad 4.490+05$

$5.760+021.986+06$

$6.250+021.710+06$

$8.000+021.209+06$
$4.026+028.514+05$

$4.138+028.190+05$

4. $218+021.195+06$

4. $309+021.108+06$

$4.404+021.029+06$

$4.501+029.609+05$

$4.602+028.273+05$

$4.707+026.292+05$

$4.816+025.440+05$

$4.928+025.369+05$

$5.045+024.814+05$

$5.166+02 \quad 4.637+05$

$5.292+026.276+05$

$5.423+023.211+05$

$5.559+024.721+05$

$5.700+02 \quad 4.245+05$

$5.848+022.590+06$

$6.001+022.080+06$

$6.161+021.866+06$

$6.328+021.959+06$

$6.503+021.773+06$

$6.684+021.689+06$

$6.875+02 \quad 1.703+06$

$7.075+021.728+06$

$7.283+021 \cdot 570+06$

$7.502+021.564+06$

$7.732+021.490+06$

$7.973+021.354+06$

$8.227+021.302+06$

$8.494+021.235+06$

$8.776+021.120+06$

$9.073+021.033+06$

$9.387+021.442+06$

9. $719+029.931+05$

$\mathrm{eV}, \mathrm{b} / \mathrm{atom}$ pairs

$5.100+025.000+05$

$5.500+025.000+05$

$5.720+026.000+05$

$5.800+021.540+06$

$5.880+022.350+06$

$5.960+022.140+06$

$6.040+021.800+06$

$6.120+021.670+06$

$6.200+021.620+06$

$6.280+021.630+06$

$6.360+021.620+06$

$6.440+021.580+06$

$6.520+021.570+06$

$6.600+021.520+06$

$6.680+021.520+06$

$6.760+021.520+06$

$6.840+021.480+06$

$6.920+021.450+06$

7. $000+021.500+06$

$\mathrm{eV}, \mathrm{b} /$ atom pairs

15 pts

$5.500+024.317+05$

$5.800+023.583+06$

$6.500+021.468+06$

$8.500+021.053+06$

$4.054+028.028+05$

$4.167+029.430+05$

$4.240+021.172+06$

$4.332+021.091+06$

4. $428+021.012+06$

$4.526+029.847+05$

$4.628+026.673+05$

$4.734+025.706+05$

$4.843+025.376+05$

$4.957+025.189+05$

$5.075+024.684+05$

$5.197+024.591+05$

$5.324+026.972+05$

$5.456+022.195+05$

$5.594+023.913+05$

$5.737+021.277+06$

$5.885+022.562+06$

$6.041+021.988+06$

$6.202+021.850+06$

$6.371+021.872+06$

$6.547+021.756+06$

$6.731+021.658+06$

$6.924+021.744+06$

$7.126+021.684+06$

$7 \cdot 337+021 \cdot 559+06$

$7.559+021.555+06$

$7.791+021.460+06$

$8.035+021.311+06$

8. $292+021.301+06$

$8.563+021.203+06$

$8.849+021.087+06$

$9.150+021.021+06$

$9.468+029.781+05$

$9.805+029.828+05$
$.200+024.800+05$

$5.600+025.000+05$

$5.740+026.500+05$

$5.820+021.910+06$

$5.900+022.450+06$

$5.980+021 \cdot 980+06$

$6.060+021.750+06$

$6.140+021.660+06$

$6.220+021.650+06$

$6.300+021.610+06$

$6.380+021.620+06$

$6.460+021.560+06$

$6.540+021.560+06$

$6.620+021.530+06$

$6.700+021.520+06$

$6.780+021.510+06$

$6.860+021.480+06$

$6.940+021.460+06$

\section{$5.200+024.800$}

$4.082+028.447+05$

$4.196+029.330+05$

$4.263+021.149+06$

$4.356+021.067+06$

$4.452+029.938+05$

$4.551+029.343+05$

$4.654+024.573+05$

$4.761+025.478+05$

$4.871+025.307+05$

$4.986+025.098+05$

$5.105+024.764+05$

$5.228+024.375+05$

$5.357+026.219+05$

$5.490+023.065+05$

$5.629+023.604+05$

$5.773+02 \quad 2.147+06$

$5.923+022.365+06$

$6.080+021.935+06$

$6.244+021.851+06$

$6.414+021.845+06$

$6.593+021.748+06$

$6.779+021.649+06$

$6.974+021.753+06$

$7.177+021.643+06$

$7.391+021.552+06$

7. $616+021.542+06$

$7.851+021.416+06$

$8.099+021.297+06$

$8.359+021.284+06$

$8.633+021.173+06$

$8.923+029.866+05$

$9.228+029.172+05$

$9.551+021.021+06$

$9.892+029.727+05$

ref $=87 \mathrm{De} 02$

$5.300+02 \quad 4.800+05$

$5.650+02 \quad 5.200+05$

$5.760+028.900+05$

$5.840+02 \quad 2.180+06$

$5.920+022.390+06$

$6.000+021.880+06$

$6.080+021.700+06$

$6.160+021.640+06$

$6.240+021.620+06$

$6.320+021.630+06$

$6.400+021.620+06$

$6.480+021.560+06$

$6.560+021.550+06$

$6.640+021.540+06$

$6.720+021.540+06$

$6.800+021.500+06$

$6.880+021.480+06$

$6.960+021.450+06$

ref $=90$ De01

$5.730+023.575+06$

$6.000+021.770+06$

$7.500+021.347+06$ 
$\mathrm{Z}=26, \mathrm{Fe}$

$6.000+025.101+05$

$7.020+024.451+06$

$7.500+021.298+06$

$9.500+029.227+05$

$Z=26, \mathrm{Fe}$

1. $000+038.321+05$

3. $000+035.408+04$

$5.947+03 \quad 8.133+03$

$7.112+03 \quad 4.224+04$

$9.962+031.603+04$

$2.494+041.267+03$

$Z=26, \mathrm{Fe}$

$4.509+031.764+04$

$5.412+031.024+04$

$6.490+036.408+03$

$7.649+03 \quad 3.292+04$

$8.905+03 \quad 2.254+04$

$\mathrm{Z}=26, \mathrm{Fe}$

$2.260+04$ i. $641+03$

$\mathrm{Z}=26, \mathrm{Fe}$

$3.313+03 \quad 4.266+04$

$4.508+031.818+04$

$4.950+031.398+04$

$7.472+03 \quad 3.651+04$

$8.905+03 \quad 2.255+04$

1. $054+041.462+04$

1. $262+048 \cdot 940+03$

$1.517+04 \quad 5.407+03$

$1.895+042.829+03$

$Z=26, \mathrm{Fe}$

$7.280+062.800+00$

$\mathrm{Z}=27$, Co

$4.509+03 \quad 2.033+04$

5. $412+031.227+04$

$6.490+037.428+03$

$7.649+034.756+03$

$8.905+032.515+04$

$\mathrm{Z}=28, \mathrm{Ni}$

2. $045+02 \quad 2.673+06$

$2.084+02 \quad 2.640+06$

$2.124+022.612+06$

2. $165+022.553+06$

2. $208+022.481+06$

2. $251+022.422+06$

$2.297+02 \quad 2.352+06$

$2.343+02 \quad 2.273+06$

$2.391+022.217+06$

$2.441+02 \quad 2.154+06$

$2.492+022.183+06$

$2.544+021.992+06$

$2.599+02 \quad 1.897+06$

$2.655+021.827+06$

$2.714+021.712+06$

$2.774+021.644+06$

eV, b/atom pairs

16 pts

$6.500+024.637+05$

$7.000+024.173+05$

$7.120+021.113+06$

$7.180+02 \quad 2.272+06$

$8.000+021.159+06$

$8.500+021.113+06$

$1.000+037.419+05$

1. $050+03$

$6.955+05$

eV, b/atom pairs

$1.487+03 \quad 3.409+05$

$4.000+032.395+05$

$6.000+037.869+03$

$7.472+03 \quad 3.570+04$

$1.000+041.597+04$

3. $000+047.572+02$

eV, b/atom pairs

$4.932+031.391+04$

$5.895+038.189+03$

$6.925+035.184+03$

$8.041+032.860+04$

$9.572+031.790+04$

eV, b/atom pairs

$1.500+033.340+05$

$5.000+031.280+04$

$6.400+036.375+03$

$8.000+032.908+04$

$1.500+045.305+03$

$4.000+043.342+02$

$4.950+031.311+04$

$5.945+038.031+03$

$7.058+035.147+03$

$8.265+032.671+04$

$9.876+031.651+04$

eV, b/atom pairs

35 pts

$3.590+03 \quad 3.496+04$

$4.509+031.812+04$

$5.427+031.099+04$

$8.041+032.986+04$

$9.185+032.124+04$

1. $121+041.294+04$

$1.338+047.540+03$

$1.658+04 \quad 4.229+03$

$1.960+042.624+03$

eV, b/atom pairs

$3.937+032.652+04$

4. $802+031.530+04$

$6.399+036.408+03$

$8.265+032.762+04$

$9.707+031.819+04$

1. $135+041.187+04$

$1.377+046.770+03$

1. $744+043.672+03$

$1.997+042.522+03$

$4.950+031.561+04$ $4.932+031.536+04$

$5.895+039.630+03$

$6.925+036.146+03$

$8.041+03 \quad 3.397+04$

$5.945+039.297+03$

$7.058+035.852+03$

$8.265+03 \quad 3.102+04$

$9.572+032.075+04$

$9.876+03$

1. $888+04$

eV, b/atom pairs

256 pts

$2.055+022.674+06$

$2.094+022.630+06$

$2.134+022.602+06$

2. $176+02 \quad 2.538+06$

2. $219+02 \quad 2.461+06$

$2.263+022.405+06$

$2.308+022.336+06$

2. $355+022.264+06$

$2.403+022.193+06$

2. $453+022.135+06$

2. $505+022.060+06$

$2.558+021.965+06$

$2.613+021.858+06$

$2.670+021.785+06$

$2.728+021.708+06$

$2.789+021.615+06$
$2.064+022.666+06$

$2.104+022.621+06$

$2.144+022.580+06$

$2.186+022.516+06$

2. $229+022.451+06$

$2.274+022.384+06$

$2.320+022.320+06$

2. $367+022.243+06$

$2.416+022.182+06$

$2.466+022.116+06$

$2.518+022.044+06$

$2.571+021.945+06$

$2.627+021.829+06$

$2.684+021.747+.06$

$2.743+021.687+06$

$2.805+021.603+06$ ref $=90 \mathrm{De} 01$

$7.010+021.855+06$

$7.250+021.159+06$

$9.000+021.020+06$

$1.100+036.492+05$

ref $=86 \mathrm{De} 02$

$2.000+031.590+05$

$5.415+03 \quad 1.036+04$

$7.058+034.913+03$

$8.398+032.578+04$

$2.000+04 \quad 2.379+03$

ref $=89$ Uno1

$5.427+03 \quad 1.056+04$

$6.400+036.584+03$

$7.476+03 \quad 3.634+04$

$8.631+032.349+04$

1. $098+041.271+04$

ref $=90 Q u 01$

ref $=92 \mathrm{WaOI}$

$4.251+032.152+04$

$4.932+031.402+04$

$7.058+03 \quad 4.841+03$

$8.494+03 \quad 2.587+04$

$1.031+041.516+04$

$1.148+041.159+04$

$1.476+045.787+03$

$1.862+043.042+03$

ref $=84 \mathrm{YeOI}$

ref $=89 \mathrm{Un} 01$

$5.427+03 \quad 1.228+04$

$6.400+037.604+03$

$7.476+035.079+03$

$8.631+032.706+04$

1. $098+041.415+04$

ref $=90 \mathrm{Fu} 01$

$2.074+022.653+06$

2. $114+022.609+06$

$2.155+022.562+06$

$2.197+022.496+06$

$2.240+022.438+06$

$2.285+02 \quad 2.370+06$

$2.331+022.298+06$

2. $379+022.229+06$

$2.428+022.174+06$

$2.479+022.101+06$

$2.531+022.014+06$

$2.585+021.919+06$

$2.641+021.798+06$

$2.699+021.715+06$

$2.759+021.660+06$

$2.820+021.503+06$ 
$2.836+021.288+06$

$2.901+021.030+06$

$2.967+027.751+05$

3. $037+029.965+05$

3.108+02 1.155+06

$3.183+021.120+06$

$3.260+021.077+06$

$3.340+031.065+06$

3. $424+021.026+06$

$3.510+029.591+05$

$3.600+029.166+05$

$3.694+028.650+05$

$3.791+028.116+05$

$3.893+027.601+05$

$3.999+026.824+05$

4.110+02 5.005+05

$4.196+022.013+05$

$4.286+028.068+05$

$4.380+029.041+05$

$4.476+028.854+05$

$4.577+028.622+05$

$4.681+026.720+05$

$4.788+025.384+05$

$4.900+025.029+05$

$5.015+024.733+05$

$5.135+024.735+05$

$5.260+023.300+05$

$5.390+023.296+05$

$5.524+023.468+05$

$5.664+023.154+05$

$5.810+022.740+05$

$5.962+023.048+05$

$6.121+023.117+05$

$6.286+022.978+05$

$6.458+022.700+05$

$6.638+022.490+05$

$6.827+022.305+05$

$7.024+02 \quad 2.193+05$

$7.230+022.038+05$

$7.446+021.964+05$

$7.673+021.724+05$

$7.912+021.677+05$

8. $163+021.454+05$

$8.426+023.987+05$

$8.704+029.474+05$

$8.998+029.764+05$

$9.307+021.989+05$

$9.635+029.650+05$

$\mathrm{Z}=28, \mathrm{Ni}$

$7.500+023.606+05$

$8.560+021.910+06$

$9.999+021.082+06$

$1.050+039.350+05$

$1.250+036.042+05$

$\mathrm{Z}=28, \mathrm{Ni}$

$8.000+022.300+05$ $8.200+022.500+05$ $8.400+022.500+05$ $8.480+023.200+05$ $8.560+024.700+05$
$2.852+021.390+06$

2. $917+028.517+05$

2. $985+027.846+05$

3. $054+021.074+06$

3. $127+02$ 1. $140+06$

3. $202+021.120+06$

3. $280+021.073+06$

$3.361+021.052+06$

3. $445+021.009+06$

$3.532+029.430+05$

$3.623+029.041+05$

3. $718+028.519+05$

3. $816+027.954+05$

3. $919+027.502+05$

$4.026+026.557+05$

$4.138+023.859+05$

4. $218+023.762+05$

$4.309+028.559+05$

$4.404+028.901+05$

$4.501+028.921+05$

$4.602+029.682+05$

$4.707+026.030+05$

4. $816+025.296+05$

$4.928+024.871+05$

$5.045+024.732+05$

5. $166+024.377+05$

$5.292+020.000+00$

$5.423+025.907+05$

$5.559+022.307+05$

$5.700+024.718+05$

$5.848+022.665+05$

$6.001+022.858+05$

$6.161+023.079+05$

$6.328+022.972+05$

$6.503+022.684+05$

$6.684+022.504+05$

$6.875+022.271+05$

$7.075+02 \quad 2.153+05$

$7.283+021.985+05$

$7.502+021.900+05$

$7.732+02 \quad 1.677+05$

$7.973+021.628+05$

$8.227+021.426+05$

$8.494+025.976+05$

$8.776+029.681+05$

$9.073+021.004+06$

$9.387+028.734+05$

$9.719+027.223+05$
$2.868+021.192+06$

$2.934+027.611+05$

$3.002+028.155+05$

3. $072+021.136+06$

3. $145+021.137+06$

$3.221+021.088+06$

3. $300+021.063+06$

$3.381+021.049+06$

$3.466+029.859+05$

$3.555+029.347+05$

$3.647+028.983+05$

$3.742+028.566+05$

$3.842+027.802+05$

$3.945+027.361+05$

$4.054+023.645+05$

$4.167+023.545+05$

$4.240+026.513+05$

$4.332+029.355+05$

$4.428+028.834+05$

$4.526+028.903+05$

$4.628+021.020+06$

$4.734+026.043+05$

$4.843+025.325+05$

$4.957+024.800+05$

$5.075+024.711+05$

$5.197+024.344+05$

$5.324+020.000+00$

$5.456+027.503+05$

$5.594+022.454+05$

$5.737+024.358+05$

$5.885+023.171+05$

$6.041+022.880+05$

$6.202+023.012+05$

$6.371+022.909+05$

$6.547+022.608+05$

$6.731+022.459+05$

$6.924+022.261+05$

$7.126+02 \quad 2.114+05$

$7.337+022.013+05$

$7.559+021.902+05$

$7.791+021.694+05$

$8.035+021.592+05$

$8.292+021.553+05$

$8.563+027.941+05$

$8.849+029.642+05$

9. $150+021.010+06$

$9.468+028.383+05$

$9.805+026.702+05$

eV, b/atom pairs

$8.000+022.934+05$

$8.650+02 \quad 8.674+05$

$9.500+021.004+06$

$1.100+038.479+05$

$8.500+022.144+05$

$8.750+021.511+06$

$1.000+039.551+05$

$1.150+03 \quad 7.504+05$
$2.884+021.125+06$

$2.950+027.469+05$

3. $019+028.853+05$

3. $090+021.143+06$

3. $164+021.127+06$

3. $241+021.094+06$

$3.320+021.063+06$

$3.402+021.036+06$

$3.488+029.709+05$

$3.577+029.202+05$

$3.670+028.814+05$

$3.767+028.463+05$

3. $867+027.580+05$

$3.972+026.969+05$

$4.082+024.965+05$

$4.196+023.314+05$

$4.263+028.098+05$

$4.356+029.310+05$

$4.452+028.786+05$

$4.551+028.831+05$

$4.654+029.259+05$

$4.761+025.780+05$

$4.871+025.125+05$

$4.986+024.759+05$

5. $105+024.641+05$

5. $228+024.262+05$

$5.357+024.395+04$

$5.490+025.441+05$

$5.629+022.914+05$

$5.773+022.763+05$

$5.923+023.200+05$

$6.080+023.079+05$

$6.244+022.989+05$

$6.414+022.776+05$

$6.593+022.562+05$

$6.779+022.457+05$

$6.974+022.209+05$

7. $177+022.109+05$

7. $391+021.960+05$

$7.616+021.783+05$

$7.851+021.624+05$

$8.099+021.540+05$

$8.359+022.322+05$

$8.633+029.189+05$

$8.923+029.626+05$

$9.228+021.540+06$

$9.551+027.585+05$

$9.892+026.775+05$

ref $=90 \mathrm{De} 01$

$8.530+029.746+05$

$8.850+021.072+06$

$1.025+031.004+06$

$1.200+036.725+05$
eV, b/atom pairs

$8.050+02 \quad 2.400+05 \quad 8.100+02 \quad 2.400+05$

$8.250+022.450+05$

$8.420+022.400+05$

$8.500+02 \quad 3.500+05$

$8.580+02 \quad 5.150+05$
$8.300+022.500+05$

$8.440+02 \quad 2.900+05$

$8.520+02 \quad 3.700+05$

$8.600+02 \quad 5.600+05$ ref $=87 \mathrm{De} 02$

$8.150+022.450+05$

$8.350+022.600+05$

$8.460+022.900+05$

$8.540+024.150+05$

$8.620+025.700+05$ 
$8.640+026.500+05$

$8.720+027.400+05$

$8.800+027.900+05$

$8.880+028.300+05$

$8.960+028.400+05$

9. $040+028.700+05$

$9.120+029.000+05$

$9.200+028.800+05$

$9.280+028.700+05$

9.360+02 $8.500+05$

$9.440+027.900+05$

$9.520+027.600+05$

$9.600+027.700+05$

$9.680+027.400+05$

$9.760+027.300+05$

$9.840+027.200+05$

$9.920+027.200+05$

$1.000+036.900+05$

$\mathrm{Z}=28, \mathrm{Ni}$

1. $487+034.492+05$

$4.000+03 \quad 3.232+04$

$6.000+031.062+04$

$8.333+033.528+04$

$\mathrm{Z}=28, \mathrm{Ni}$

$4.509+03 \quad 2.471+04$

$5.412+031.452+04$

$6.490+039.034+03$

$7.649+035.380+03$

$8.905+032.943+04$

$\mathrm{Z}=28, \mathrm{Ni}$

$8.332+031.975+04$

$\mathrm{Z}=28, \mathrm{Ni}$

5. $240+042.000+02$

$\mathrm{Z}=28, \mathrm{Ni}$

$7.280+063.090+00$

$\mathrm{Z}=29, \mathrm{Cu}$

8. $000+022.533+05$

$9.550+026.226+05$

1. $050+039.286+05$

$1.300+036.595+05$

$\mathrm{Z}=29, \mathrm{Cu}$

$8.400+022.800+05$

$8.600+022.750+05$

$8.800+022.800+05$

$9.000+022.900+05$

$9.080+022.800+05$

9. $160+022.800+05$

$9.240+023.100+05$

$9.320+023.500+05$

$9.400+024.300+05$

$9.480+024.950+05$

$9.560+025.500+05$

$9.640+026.100+05$

$9.720+026.300+05$

$9.800+026.500+05$
$8.660+026.800+05$

$8.740+027.500+05$

$8.820+028.000+05$

$8.900+028.100+05$

$8.980+028.400+05$

9. $060+028.900+05$

9. $140+029.000+05$

$9.220+028.900+05$

$9.300+028.600+05$

$9.380+028.600+05$

$9.460+027.900+05$

$9.540+027.650+05$

$9.620+027.650+05$

$9.700+027.300+05$

$9.780+027.200+05$

$9.860+026.900+05$

$9.940+027.300+05$
$8.680+027.000+05$

8. $760+027.700+05$

$8.840+028.150+05$

$8.920+028.300+05$

$9.000+028.500+05$

$9.080+029.000+05$

9. $160+028.900+05$

$9.240+028.900+05$

$9.320+028.700+05$

$9.400+028.300+05$

$9.480+028.000+05$

$9.560+027.700+05$

$9.640+027.550+05$

$9.720+027.300+05$

$9.800+026.900+05$

$9.880+026.900+05$

$9.960+026.900+05$

16 pts

$1.500+03 \quad 4.326+05 \quad 2.000+03 \quad 2.132+05$

$5.000+03 \quad 1.731+04 \quad 5.415+03 \quad 1.400+04$

$7.472+03 \quad 5.763+03 \quad 8.000+03 \quad 4.716+03$

$1.000+042.082+04 \quad 1.500+046.982+03$

$\mathrm{eV}, \mathrm{b} / \mathrm{atom}$ pairs

$4.932+03 \quad 1.842+04 \quad 4.950+03 \quad 1.895+04$

$5.895+03 \quad 1.176+04 \quad 5.945+03 \quad 1.131+04$

$6.925+037.241+03 \quad 7.058+036.978+03$

$8.041+03 \quad 4.775+03 \quad 8.265+03 \quad 4.522+03$

$9.572+03 \quad 2.436+04 \quad 9.876+03 \quad 2.191+04$

$\mathrm{eV}, \mathrm{b} / \mathrm{atom}$ pairs

$8.361+03 \quad 3.216+04 \quad 9.000+03$

eV, b/atom pairs

eV, b/atom pairs

$\mathrm{eV}, \mathrm{b} / \mathrm{atom}$ pairs

9. $000+021.794+05$

$9.700+029.233+05$

$1.085+038.442+05$

$9.200+021.583+05$

$1.000+039.919+05$

1. $100+038.442+05$
$8.700+027.300+05$

$8.780+027.800+05$

$8.860+028.300+05$

$8.940+028.400+05$

$9.020+028.700+05$

9. $100+029.000+05$

$9.180+028.800+05$

$9.260+028.700+05$

$9.340+028.550+05$

9. $420+028.000+05$

$9.500+027.700+05$

9. $580+027.700+05$

$9.660+027.550+05$

$9.740+027.100+05$

$9.820+026.900+05$

$9.900+027.000+05$

$9.980+026.800+05$ ref $=86 \mathrm{De} 02$

$3.000+037.175+04$

$5.947+031.084+04$

$8.245+034.355+03$

$2.000+043.169+03$

ref $=89 \mathrm{UnO} 01$

$5.427+03 \quad 1.476+04$

$6.400+039.103+03$

$7.476+035.818+03$

$8.631+03 \quad 3.296+04$

$1.098+041.657+04$

ref $=88 \mathrm{PaO} 1$

ref $=89 \mathrm{Ke} 01$

ref $=84 \mathrm{Ye} 01$

ref $=90 \mathrm{De} 01$

$9.450+026.964+05$

$1.020+03 \quad 9.286+05$

$1.200+03 \quad 8.125+05$
eV, b/atom pairs

$8.450+022.900+05$

$8.650+02 \quad 2.700+05$

$8.850+02 \quad 2.800+05$

$9.020+022.800+05$

$9.100+02 \quad 2.900+05$

$9.180+02 \quad 3.000+05$

$9.260+02 \quad 3.300+05$

$9.340+02 \quad 3.800+05$

$9.420+02 \quad 4.300+05$

$9.500+02 \quad 5.300+05$

$9.580+02 \quad 5.700+05$

$9.660+02 \quad 6.200+05$

$9.740+026.400+05$

$9.820+026.750+05$
63 pts

$8.500+022.750+05$

$8.700+022.700+05$

$8.900+02 \quad 2.700+05$

$9.040+022.700+05$

$9.120+022.850+05$

$9.200+023.150+05$

$9.280+02 \quad 3.400+05$

$9.360+023.900+05$

$9.440+024.500+05$

$9.520+025.400+05$

$9.600+025.800+05$

$9.680+026.300+05$

$9.760+026.500+05$

$9.840+026.850+05$ ref $=87 \mathrm{De} 02$

$8.550+02 \quad 2.700+05$

$8.750+022.750+05$

$8.950+02 \quad 2.800+05$

$9.060+022.800+05$

$9.140+023.000+05$

$9.220+02 \quad 3.100+05$

$9.300+023.500+05$

$9.380+024.000+05$

$9.460+02 \quad 4.950+05$

$9.540+02 \quad 5.550+05$

$9.620+026.000+05$

$9.700+026.300+05$

$9.780+02 \quad 6.600+05$

$9.860+026.800+05$ 
$9.880+026.800+05$

$9.960+026.800+05$

$\mathrm{Z}=29, \mathrm{Cu}$

$3.313+036.791+04$

$4.508+03 \quad 2.902+04$

$4.950+03 \quad 2.211+04$

$7.472+037.038+03$

$8.905+03 \quad 4.337+03$

$1.054+04 \quad 2.047+04$

$1.262+04 \quad 1.258+04$

$1.476+04 \quad 8.273+03$

$1.862+04 \quad 4.411+03$

$2.210+042.817+03$

$2.847+04 \quad 1.446+03$

$\mathrm{Z}=29, \mathrm{Cu}$

$4.509+03 \quad 2.787+04$

$5.412+03 \quad 1.667+04$

$6.490+031.006+04$

$7.649+03 \quad 6.321+03$

$8.905+03 \quad 4.136+03$

$\mathrm{Z}=29, \mathrm{Cu}$

$5.893+03 \quad 1.200+04$

$\mathrm{Z}=29, \mathrm{Cu}$

$5.895+03 \quad 1.274+04$

$\mathrm{Z}=29, \mathrm{Cu}$

$5.947+03 \quad 1.253+04$

$\mathrm{z}=29, \mathrm{Cu}$

$6.400+03 \quad 1.015+04$

$\mathrm{Z}=29, \mathrm{Cu}$

$6.460+031.009+04$

$\mathrm{Z}=29, \mathrm{Cu}$

$8.118+03 \quad 5.371+03$

$\mathrm{Z}=29, \mathrm{Cu}$

$1.343+04 \quad 1.097+04$

$5.920+041.698+02$

$\mathrm{Z}=29, \mathrm{Cu}$

$1.390+04 \quad 1.042+04$

$5.950+041.636+02$

$\mathrm{z}=29, \mathrm{Cu}$

$3.080+041.060+03$

$3.030+051.160+01$

$\mathrm{Z}=29, \mathrm{Cu}$

$4.300+04 \quad 4.137+02$

$9.900+04 \quad 4.905+01$

$z=29, \mathrm{Cu}$

$5.240+04 \quad 2.280+02$

$\mathrm{Z}=29, \mathrm{Cu}$

$7.962+048.000+01$
$9.900+02 \quad 6.830+05$

$9.980+026.900+05$

$9.920+026.900+05$

$1.000+036.930+05$

$\mathrm{eV}, \mathrm{b} / \mathrm{atom}$ pairs

3. $590+03 \quad 5.253+04$

42 pts

$4.509+03 \quad 2.817+04$

$5.427+03 \quad 1.682+04$

$8.041+03 \quad 5.666+03$

$9.185+03 \quad 3.481+04$

$1.135+041.813+04$

$1.302+041.132+04$

1. $517+04 \quad 7.292+03$

$1.895+04 \quad 4.295+03$

$2.493+04 \quad 1.984+03$

$2.911+041.393+03$

$3.937+03 \quad 4.146+04$

$4.802+03 \quad 2.427+04$

$6.399+031.053+04$

$8.265+03 \quad 5.297+03$

$9.707+03 \quad 2.560+04$

$1.148+04 \quad 1.597+04$

$1.338+04 \quad 1.062+04$

$1.658+046.025+03$

$1.960+043.894+03$

$2.519+04 \quad 1.931+03$

$\mathrm{eV}, \mathrm{b} / \mathrm{atom}$ pairs

$4.932+032.237+04$

$5.895+03 \quad 1.318+04$

$6.925+03 \quad 8.421+03$

$8.041+03 \quad 5.498+03$

$9.572+03 \quad 2.754+04$

$4.950+03 \quad 2.167+04$

$5.945+03 \quad 1.283+04$

$7.058+03 \quad 8.305+03$

$8.265+03 \quad 5.065+03$

$9.876+03 \quad 2.418+04$

eV, b/atom pairs

4 pts

$1.337+04 \quad 1.010+04 \quad 1.744+04 \quad 5.000+03$

$\mathrm{eV}, \mathrm{b} / \mathrm{atom}$ pairs

$8.041+03 \quad 5.496+03$

eV, b/atom pairs

2 pts

1 pts

$\mathrm{eV}, \mathrm{b} / \mathrm{atom}$ pairs

1 pts

eV, b/atom pairs

1 pts

$\mathrm{eV}, \mathrm{b} /$ atom pairs

1 pts

5 pts

eV, b/atom pairs

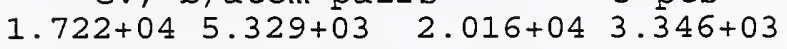

$\mathrm{eV}, \mathrm{b} / \mathrm{atom}$ pairs

$\begin{array}{lll}1.780+04 & 5.255+03 & 2.630+04\end{array}$

5 pts

$1.688+03$

$\mathrm{eV}, \mathrm{b} / \mathrm{atom}$ pairs

7 pts

$7.800+01$

$3.500+04 \quad 7.720+02$

$8.100+04$

$9.900+00$

$\mathrm{eV}, \mathrm{b} / \mathrm{atom}$ pairs

7 pts

$5.300+04 \quad 2.388+02 \quad 5.950+04 \quad 1.701+02$

$1.220+053.295+01 \quad 1.520+052.263+01$

eV, b/atom pairs

1 pts

3 pts

$\mathrm{eV}, \mathrm{b} / \mathrm{atom}$ pairs

$8.100+047.730+018.091+04 \quad 7.7400+01$

\section{$9.940+026.850+05$}

ref $=92 \mathrm{Wa01}$

$4.251+03 \quad 3.329+04$

$4.932+03 \quad 2.247+04$

$7.058+03 \quad 7.935+03$

$8.494+03 \quad 4.780+03$

$1.031+042.173+04$

$1.192+041.378+04$

$1.377+04 \quad 9.814+03$

$1.744+04 \quad 5.403+03$

$1.997+043.714+03$

$2.546+04 \quad 1.773+03$

ref $=89 \mathrm{Un} 01$

$5.427+03 \quad 1.684+04$

$6.400+03 \quad 1.065+04$

$7.476+03 \quad 6.753+03$

$8.631+03 \quad 4.474+03$

$1.098+041.847+04$

ref $=88 \mathrm{NaOl}$

$2.016+042.850+03$

ref $=94 \mathrm{KeOI}$

ref $=91 \mathrm{Ke} 01$

ref $=93 \mathrm{Ke} 02$

ref $=93 \mathrm{Ke} 01$

ref $=93 \mathrm{Ke} 03$

ref $=84 \mathrm{NaO} 01$

$4.652+04 \quad 3.324+02$

ref $=95 \mathrm{Ta} 01$

$3.320+048.526+02$

ref $=93 \mathrm{NaO} 1$

$2.760+051.220+01$

ref $=87 \mathrm{MaO} 1$

$8.100+04 \quad 7.798+01$

ref $=89 \mathrm{Ke} 01$

ref $=87$ Re01 
$\mathrm{Z}=30, \mathrm{Zn}$

$4.509+03 \quad 3.142+04$

$5.412+031.889+04$

$6.490+031.153+04$

$7.649+037.155+03$

$8.905+034.636+03$

$\mathrm{Z}=30, \mathrm{Zn}$

2. $260+04 \quad 3.822+03$

$\mathrm{Z}=30, \mathrm{Zn}$

$4.300+04 \quad 4.734+02$

$9.900+045.450+01$

$\mathrm{Z}=36, \mathrm{Kr}$

$1.667+01 \quad 4.490+07$

$\mathrm{Z}=36, \mathrm{Kr}$ (K-edge)

$1.425+041.913+03$

$1.429+04 \quad 2.099+03$

$1.433+049.896+03$

$1.437+041.954+04$

$1.441+041.890+04$

$1.445+041.873+04$

1. $449+041.864+04$

$1.453+04 \quad 1.851+04$

$1.843+04 \quad 1.457+04$

$1.460+04 \quad 1.833+04$

$1.464+041.825+04$

$1.468+041 \cdot 813+04$

1. $472+041.804+04$

$Z=39, Y$

3. $313+032.045+05$

$4.590+037.052+04$

$7.472+03 \quad 2.293+04$

$9.185+031.314+04$

$1.262+045 \cdot 507+03$

$1.517+043.351+03$

1. $744+041.522+04$

$1.997+04 \quad 1.033+04$

2. $545+045.389+03$

$\mathrm{Z}=40, \mathrm{Zr}$

1. $390+04 \quad 4.604+03$

$5.950+045.589+02$

$\mathrm{Z}=41, \mathrm{Nb}$

1. $390+04 \quad 4.752+03$

$5.950+046.233+02$

$\mathrm{Z}=42$, Mo

$6.400+034.591+04$

$\mathrm{Z}=42$, Mo

$6.460+03 \quad 4.432+04$

$Z=42$, Mo

$1.390+045.656+03$

$5.950+046.962+02$
$\mathrm{eV}, \mathrm{b} /$ atom pairs

20 pts

$4.932+03 \cdot 2.464+04 \quad 4.950+03 \quad 2.419+04$

$5.895+031.495+04$

$5.945+031.460+04$

$6.925+039.576+03$

$8.041+036.308+03$

$9.572+03 \quad 4.245+03$

$7.058+03$

$8.265+03$

$9.876+03$

$9.207+03$

$5.808+03$

eV, b/atom pairs

1 pts

$\mathrm{eV}, \mathrm{b} / \mathrm{atom}$ pairs

7 pts

$\begin{array}{llll}5.300+04 & 2.655+02 & 5.950+04 & 1.680+02\end{array}$

$1.220+053.600+01 \quad 1.520+05$

$2.445+01$

eV, b/atom pairs

$\begin{array}{lllll}1.685+01 & 4.487+07 & 2.122+01 & 3.831+07\end{array}$

$\mathrm{eV}, \mathrm{b} / \mathrm{atom}$ pairs

50 pts

$1.426+04 \quad 1.956+03 \quad 1.427+04 \quad 2.037+03$

1. $430+042 \cdot 163+03$

1. $431+042 \cdot 347+03$

1. $434+041 \cdot 973+04$

$1.435+04$

$1.438+04 \quad 1 \cdot 935+04$

$1.442+041.884+04$

1. $439+04$

$1.986+04$
$1.917+04$

1. $446+04 \quad 1.872+04$

$1.450+041.859+04$

1. $443+04$

1. $447+04$

447+04 $1.871+04$

1. $454+04 \quad 1.847+04$

$.451+041.859+04$

1. $455+04$ 1. $847+04$

$\begin{array}{llll}1.841+04 & 1.458+04 & 1.837+04 & 1.459+04\end{array}$

$1.461+041.831+04$

$\begin{array}{rr}1.837+04 & 1.459+04 \\ 1.462+04 & 1.828+04\end{array}$

$1.465+04 \quad 1 \cdot 823+04$

$1.466+041.819+04$

$1.469+041.812+04$

$1.470+041 \cdot 809+04$

$1.473+041 \cdot 804+04$

$1.474+04$

$1.800+04$

eV, b/atom pairs

3. $590+031.652+05$

35 pts

$5.427+035.467+04$

$4.509+039.136+04$

$6.399+03 \quad 3.617+04$

$8.041+031.940+04$

1. $054+049.109+03$

$8.265+031.721+04$

$1.302+045.079+03$

$1.214+046.053+03$

$1.658+042.635+03$

1. $476+04$

1. $673+04$

$3.528+03$

1. $862+04 \quad 1.215+03$

$1.895+041.137+04$

$2.210+047.752+03$

$2.493+04$

2. $911+04$

$2.847+043.974+03$

$\mathrm{eV}, \mathrm{b} / \mathrm{atom}$ pairs

$\begin{array}{lll}1.780+04 & 2.272+03 & 2.630+04\end{array}$

5 pts

$5.498+03$

$\mathrm{eV}, \mathrm{b} / \mathrm{atom}$ pairs

5 pts

$\begin{array}{llll}1.780+04 & 2.638+03 & 2.630+04 & 6.109+03\end{array}$

$\mathrm{eV}, \mathrm{b} / \mathrm{atom}$ pairs

1 pts

ev, b/atom pairs

1 pts

5 pts

$\mathrm{eV}, \mathrm{b} / \mathrm{atom}$ pairs

$\begin{array}{lll}1.780+04 & 2.947+03 & 2.630+04\end{array}$

$6.516+03$ ref $=89 \mathrm{Un} 01$

$5.427+031.909+04$

$6.400+03 \quad 1.194+04$

$7.476+037.849+03$

$8.631+035.352+03$

1. $098+042.041+04$

ref $=90$ Qu01

ref $=87 \mathrm{MaO1}$

$8.100+048.820+01$

ref $=895 a 01$

ref $=86 \mathrm{De} 01$

1. $428+042.013+03$

1. $432+043.077+03$

$1.436+041.962+04$

1. $440+041.906+04$

1. $444+04 \quad 1.872+04$

$1.448+041.867+04$

$1.452+041.855+04$

1. $456+04$

1. $835+04$

$1.463+041.827+04$

1. $467+041.815+04$

$1.471+041.807+04$

ref $=92 \mathrm{WaO} 2$

$4.932+037.073+04$

$7.058+032.817+04$

$8.905+03 \quad 1.442+04$

$1.231+045.654+03$

$1.493+043.422+03$

1. $702+042.972+03$

$1.960+041.064+04$

2. $519+045.443+03$

ref $=95 \mathrm{TaO} 1$

3. $320+042.908+03$

ref $=95 \mathrm{Ta01}$

3. $320+043.055+03$

ref $=93 \mathrm{KeO} 2$

ref $=93 \mathrm{KeOI}$

ref $=95 \mathrm{TaO1}$

$3.320+043.505+03$ 
$\mathrm{Z}=46, \mathrm{Pd}$

$1.390+047.299+03$

$5.950+049.755+02$

$\mathrm{Z}=46, \mathrm{Pd}$

1. $750+04 \quad 4.400+03$

$2.495+049.985+03$

$4.145+042.642+03$

$\mathrm{Z}=46, \mathrm{Pd}$

9. $361+042.840+02$

$\mathrm{Z}=46, \mathrm{Pd}$

1. $098+051.880+02$

$\mathrm{Z}=47, \mathrm{Ag}$

$5.893+038.580+04$

$\mathrm{Z}=47, \mathrm{Ag}$

$5.947+038.768+04$

$\mathrm{Z}=47, \mathrm{Ag}$

1. $390+047.935+03$

$5.950+049.189+02$

$\mathrm{Z}=47, \mathrm{Ag}$

1. $725+044.854+03$

$3.090+046.198+03$

$\mathrm{Z}=47, \mathrm{Ag}$

$4.300+041.656+03$

$9.900+042.660+02$

$\mathrm{Z}=47, \mathrm{Ag}$

5. $240+041.496+03$

$\mathrm{Z}=47, \mathrm{Ag}$

1. $236+051.612+02$

$\mathrm{Z}=48, \mathrm{Cd}$

1. $390+049.352+03$

$5.950+041.098+03$

$\mathrm{Z}=48, \mathrm{Cd}$

$1.735+045.226+03$

$2.485+041.960+03$

$4.000+043.248+03$

$\mathrm{Z}=48, \mathrm{Cd}$

2. $260+042.595+03$

$\mathrm{Z}=48, \mathrm{Cd}$

1. $221+051.700+02$

$\mathrm{Z}=48, \mathrm{Cd}$

$7.280+06 \quad 6.720+00$

$\mathrm{Z}=49, \mathrm{In}$

$2.622+031.428+05$

$4.509+032.034+05$

$6.399+038.522+04$

$8.265+03 \quad 4.464+04$
$\mathrm{eV}, \mathrm{b} / \mathrm{atom}$ pairs

5 pts

$1.780+04 \quad 4.347+03 \quad 2.630+04 \quad 8.695+03$

$\mathrm{eV}, \mathrm{b} / \mathrm{atom}$ pairs

9 pts

$1.970+04 \quad 3.216+03 \quad 2.080+04 \quad 2.757+03$

$2.735+04 \quad 7.864+03 \quad 3.110+04 \quad 5.735+03$

eV, b/atom pairs

$1 \mathrm{pt}$

2 pts

4 pts

eV, b/atom pairs

$4.850+03$

$1.337+048.850+03 \quad 1.744+04$

1 pts

eV, b/atom pairs

5 pts

$\mathrm{eV}, \mathrm{b} / \mathrm{atom}$ pairs

$1.780+04 \quad 4.227+03 \quad 2.630+04 \quad 8.849+03$

$\mathrm{eV}, \mathrm{b} / \mathrm{atom}$ pairs

$\begin{array}{llll}1.980+04 & 3.466+03 & 2.480+04 & 1.845+03\end{array}$

$3.425+04 \quad 4.603+03 \quad 4.130+04$

$2.866+03$

$\mathrm{eV}, \mathrm{b} / \mathrm{atom}$ pairs

$5.300+04 \quad 1.503+03 \quad 5.950+04 \quad 1.067+03$

$1.220+051.585+02 \quad 1.520+05 \quad 9.350+01$

eV, b/atom pairs

1 pts

eV, b/atom pairs

1 pts

5 pts

$\mathrm{eV}, \mathrm{b} / \mathrm{atom}$ pairs

$1.780+04 \quad 4.797+03 \quad 2.630+04$

1. $589+03$

$\mathrm{eV}, \mathrm{b} / \mathrm{atom}$ pairs

$1.915+04 \quad 3.976+03 \quad 2.110+04 \quad 3.136+03$

$2.730+04 \quad 8.923+03 \quad 3.095+04 \quad 6.580+03$

eV, b/atom pairs

1 pts

2 pts

$1 \mathrm{pt}$

49 pts

$\mathrm{eV}, \mathrm{b} / \mathrm{atom}$ pairs

$2.816+03 \quad 1.207+05 \quad 3.313+03 \quad 8.900+04$

$4.932+03 \quad 1.737+05 \quad 4.950+03 \quad 1.755+05$

$7.058+03 \quad 6.622+04 \quad 7.472+03 \quad 5.732+04$

$8.494+03 \quad 4.149+04$
$8.905+03$ ref $=87 \mathrm{KeOI}$

$2.240+04 \quad 2.224+03$

3. $555+043.994+03$

ref $=87 \mathrm{Sa01}$

ref $=87 \mathrm{SaO} 2$

ref $=88 \mathrm{NaO1}$

$2.016+042.500+03$

ref $=91 \mathrm{KeOI}$

ref $=95 \mathrm{Ta01}$

$3.320+044.460+03$

ref $=87 \mathrm{KeOl}$

$2.735+048.329+03$

ref $=87 \mathrm{MaO} 01$

8. $100+04 \quad 4.440+02$

ref $=89 \mathrm{Ke} 01$

ref $=92 \mathrm{JaO}$

ref $=95 \mathrm{TaO} 1$

$3.320+045.320+03$

ref $=87 \mathrm{KeO}$

$2.265+04 \quad 2.464+03$

3. $445+04 \quad 4.853+03$

ref $=900 u 01$

ref $=87 \mathrm{SaO} 1$

ref $=84$ Ye01

ref $=94 \mathrm{WaO} 2$

$3.590+037.150+04$

$5.427+031.348+05$

$8.041+034.807+04$

9. $185+033.423+04$ 
$9.707+032.902+04$

1. $135+041.852+04$

$1.231+041.525+04$

$1.377+041.105+04$

$1.658+046.871+03$

1. $862+04 \quad 4.974+03$

2. $210+043.041+03$

$2.546+042.085+03$

$2.911+048.828+03$

$\mathrm{Z}=49$, In

1. $390+041.009+04$

$5.950+041.197+03$

$\mathrm{Z}=49, \mathrm{In}$

1. $580+047.169+03$

$2.730+041.735+03$

$4.275+042.936+03$

$\mathrm{Z}=50, \mathrm{Sn}$

$1.000+031.677+06$

$3.000+031.271+05$

$6.000+031.066+05$

$8.398+03 \quad 4.456+04$

1. $000+04 \quad 2.763+04$

3. $000+048.396+03$

$\mathrm{Z}=50, \mathrm{Sn}$

3. $313+039.681+04$

$4.950+031.776+05$

$7.472+036.210+04$

$9.185+03 \quad 3.674+04$

1. $262+04 \quad 1.480+04$

$1.517+048.822+03$

$1.862+045.180+03$

2. $210+043.278+03$

$2.847+041.640+03$

$\mathrm{Z}=50, \mathrm{Sn}$

1. $390+041 \cdot 149+04$

$5.950+041.273+03$

$\mathrm{Z}=50, \mathrm{Sn}$

$1.725+046.159+03$

$2.520+042.306+03$

$4.125+043.548+03$

$\mathrm{Z}=50, \mathrm{Sn}$

2. $260+042.937+03$

$\mathrm{Z}=50, \mathrm{Sn}$

5. $240+041.746+03$

$\mathrm{Z}=50, \mathrm{Sn}$

$7.280+06 \quad 7.133+00$

$Z=53, I$

1. $720+047.797+03$

$2.510+042.950+03$

$4.000+04 \quad 4.520+03$
$1.031+042.267+04$

1. $148+041.839+04$

1. $262+041 \cdot 440+04$

$1.476+049.282+03$

$1.673+046.692+03$

$1.895+04 \quad 4.816+03$

$2.414+042.330+03$

$2.726+041.654+03$

$1.054+04 \quad 2.347+04$

$1.192+041.638+04$

$1 \cdot 302+041 \cdot 322+04$

$1.493+049.016+03$

$1.702+046.232+03$

$1.960+04 \quad 4.331+03$

$2.493+042.176+03$

$2.786+041.754+03$

$\mathrm{eV}, \mathrm{b} / \mathrm{atom}$ pairs

$\begin{array}{llll}1.780+04 & 5.796+03 & 2.630+04 & 1.754+03\end{array}$

$\mathrm{eV}, \mathrm{b} / \mathrm{atom}$ pairs

9 pts

$1.980+04 \quad 3.975+03 \quad 2.180+04 \quad 3.051+03$

$2.870+04 \quad 8.675+03 \quad 3.075+04 \quad 7.055+03$

$\mathrm{eV}, \mathrm{b} / \mathrm{atom}$ pairs

$1.487+037.247+05 \quad 1.500+037.088+05$

$5.000+031.707+05 \quad 5.415+031.416+05$

$6.400+039.185+04 \quad 7.058+037.125+04$

$9.000+03 \quad 3.650+04$

9. $500+03 \quad 3.173+04$

$1.500+049.236+03$

$4.000+043.863+03$

$2.000+044.218+03$

$\mathrm{eV}, \mathrm{b} / \mathrm{atom}$ pairs

35 pts

$3.590+03 \quad 7.689+04 \quad 4.509+03 \quad 2.251+05$

$5.427+031.395+05$

$8.041+035.341+04$

1. $05.4+042.414+04$

$1.30+041.320+04$

$1.673+047.113+03$

$1.895+04 \quad 4.998+03$

$2.493+042.349+03$

$2.911+041.721+03$

$6.399+03 \quad 9.427+04$

$8.265+034.793+04$

1. $135+041.967+04$

$1.476+049.145+03$

$1.702+046.863+03$

1. $960+044.523+03$

2. $519+042.257+03$

eV, b/atom pairs

$\begin{array}{llll}1.780+04 & 5.992+03 & 2.630+04 & 1.906+03\end{array}$

ev, b/atom pairs

9 pts

$2.005+04 \quad 4.267+03 \quad 2.115+04 \quad 3.646+03$

$2.745+04 \quad 1.813+03 \quad 3.090+04$

$7.726+03$

ev, b/atom pairs

1 pts

eV, b/atom pairs

1 pts

eV, b/atom pairs

$1 \mathrm{pt}$

$\mathrm{eV}, \mathrm{b} / \mathrm{atom}$ pairs

$2.010+04 \quad 5.279+03 \quad 2.100+04 \quad 4.615+03$

$2.740+042.371+03$

3. $460+04$

$6.701+03$
$1.121+041 \cdot 920+04$

$1.214+041 \cdot 558+04$

$1.338+041.211+04$

$1.517+048.783+03$

$1.744+045.913+03$

$1.997+04 \quad 4.122+03$

$2.519+042.119+03$

2. $847+049.235+03$

ref $=95 \mathrm{Ta01}$

3. $320+045.262+03$

ref $=87 \mathrm{KeO} 01$

$2.515+042.116+03$

$3.465+045.300+03$

ref $=86 \mathrm{De} 02$

$2.000+033.577+05$

$5.947+031.101+05$

$8.000+035.065+04$

$9.962+032.791+04$

$2.494+042.284+03$

ref $=94 \mathrm{WaOl}$

$4.932+031.800+05$

$7.085+047.263+04$

$8.905+03 \quad 3.934+04$

1. $214+041.653+04$

$1.493+049.374+03$

1. $744+046.348+03$

$1.997+04 \quad 4.354+03$

$2.546+042.176+03$

ref $=95 \mathrm{TaOl}$

$3.320+046.228+03$

ref $=87 \mathrm{KeOl}$

$2.205+043.311+03$

$3.440+045.735+03$

ref $=90 Q u 01$

ref $=89 \mathrm{Ke} 01$

ref $=84 \mathrm{Ye} 01$

ref $=87 \mathrm{KeO}$

$2.295+04 \quad 3.646+03$

$3.720+04 \quad 5.500+03$ 
$\mathrm{Z}=54, \mathrm{Xe}$

$1.667+015.265+07$

$\mathrm{Z}=54, \mathrm{Xe}$

1. $730+048.241+03$

$2.925+042.071+03$
$\mathrm{eV}, \mathrm{b} / \mathrm{atom}$ pairs

$\begin{array}{llll}1.685+01 & 5.188+07 & 2.122+01 & 3.171+07\end{array}$
7 pts

$\begin{array}{llll}1.965+04 & 6.039+03 & 2.345+04 & 3.728+03\end{array}$

$3.910+04 \quad 5.145+03 \quad 4.400+04 \quad 3.750+03$
$\mathrm{Z}=54, \mathrm{Xe}$ (K-edge)

3. $300+041.354+03$

3. $304+041.355+03$

$3.308+041.350+03$

3. $311+041.346+03$

3. $315+041.344+03$

3. $319+041 \cdot 340+03$

3. $323+041.332+03$

$3.340+041.315+03$

3. $380+041.273+03$

$3.388+041.263+03$

$3.396+041.260+03$

$3.404+041.250+03$

$3.412+041.243+03$

3. $420+041.238+03$

$3.428+041.237+03$

$3.436+041.243+03$

3. $442+041.253+03$

$3.446+041.271+03$

$3.450+041.325+03$

$3.454+041.563+03$

$3.458+046.078+03$

3. $462+046.950+03$

$3.466+046.898+03$

$3.470+046.909+03$

$3.474+046.898+03$

$3.478+046.882+03$

$3.482+046.850+03$

3. $486+046.816+03$

$3.490+046.784+03$

$3.494+046.742+03$

$3.498+046.713+03$

$3.502+046.672+03$

$3.506+046.644+03$

3. $510+046.607+03$

$3.514+046.579+03$

$3.518+046.549+03$

$3.522+046.517+03$

$3.526+046.496+03$

$3.530+046.466+03$

3. $534+046.444+03$

3. $538+046.424+03$

3. $542+046.401+03$

$3.546+046.377+03$

$3.550+046.359+03$

3. $554+046.325+03$

$3.558+046.313+03$

$3.562+046.293+03$

$3.566+046.272+03$

$3.570+046.253+03$

$3.574+046.228+03$

3. $578+046.211+03$

$3.582+046.187+03$

3. $586+046.173+03$

3. $590+046.146+03$
$\mathrm{eV}, \mathrm{b} /$ atom pairs

$3.301+041.357+03$

3. $305+041 \cdot 351+03$

3. $308+041.351+03$

3. $312+041 \cdot 347+03$

3. $316+041.338+03$

3. $320+041.336+03$

3. $324+041 \cdot 330+03$

3. $350+041.302+03$

3. $382+041.269+03$

$3.390+041.263+03$

3. $398+041.254+03$

3. $406+041.252+03$

3. $414+041.245+03$

3. $422+041.242+03$

3. $430+041.237+03$

3. $438+041 \cdot 243+03$

3. $443+041.258+03$

3. $447+04 \quad 1.282+03$

3. $451+041.359+03$

$3.455+04 \quad 1.897+03$

$3.459+046.859+03$

$3.463+046.920+03$

$3.467+046.901+03$

3. $471+046.916+03$

$3.475+046.891+03$

$3.479+046.869+03$

$3.483+046.843+03$

$3.487+046.815+03$

$3.491+046.777+03$

$3.495+046.735+03$

$3.499+046.704+03$

$3.503+046.663+03$

$3.507+04 \quad 6.629+03$

$3.511+046.602+03$

3. $515+046.573+03$

3. $519+046.539+03$

$3.523+046.513+03$

$3.527+046.489+03$

$3.531+046.460+03$

3. $535+046.433+03$

$3.539+046.416+03$

$3.543+046.393+03$

$3.547+046.370+03$

$3.551+046.345+03$

$3.555+046.325+03$

$3.559+046.306+03$

$3.563+046.289+03$

$3.567+046.268+03$

$3.571+043.601+03$

$3.575+046.227+03$

$3.579+046.199+03$

$3.583+046.184+03$

$3.587+046.164+03$

3. $591+046.142+03$
458 pts

3. $302+041.354+03$

3. $306+041.348+03$

3. $309+041.348+03$

3. $313+041.343+03$

3. $317+041.336+03$

3. $321+041.334+03$

3. $325+041.330+03$

3. $360+041.291+03$

3. $384+041.269+03$

3. $392+041.260+03$

3. $400+04$ 1. $253+03$

3. $408+041.251+03$

3. $416+04$ 1. $244+03$

3. $424+041.237+03$

3. $432+041.238+03$

3. $440+041.244+03$

3. $444+042.263+03$

3. $448+041.296+03$

3. $452+041.389+03$

$3.456+042.525+03$

3. $460+046.943+03$

3. $464+046.900+03$

3. $468+046.904+03$

3. $472+046.906+03$

$3.476+046.896+03$

3. $480+046.866+03$

3. $484+046.840+03$

$3.488+046.797+03$

3. $492+046.760+03$

$3.496+046.728+03$

$3.500+046.698+03$

$3.504+046.657+03$

$3.508+046.631+03$

$3.512+046.596+03$

3. $516+046.560+03$

$3.520+046.536+03$

$3.524+046.508+03$

3. $528+046.479+03$

$3.532+046.458+03$

$3.536+046.429+03$

3. $540+046.412+03$

3. $544+046.392+03$

$3.548+046.361+03$

$3.552+046.341+03$

3. $556+046.322+03$

$3.560+046.301+03$

$3.564+046.281+03$

3. $568+046.268+03$

$3.572+046.250+03$

$3.576+046.218+03$

3. $580+046.197+03$

$3.584+046.177+03$

$3.588+046.155+03$

$3.592+046.137+03$ ref $=895001$

ref $=87 \mathrm{KeOl}$

$2.730+042.398+03$

ref $=91 \mathrm{De} 01$

$3.303+041.357+03$

3. $307+041.350+03$

3. $310+041.351+03$

3. $314+041.342+03$

3. $318+041.340+03$

3. $322+041.331+03$

3. $330+041.325+03$

3. $370+041.283+03$

3. $386+041.268+03$

3. $394+041.258+03$

3. $402+041.255+03$

3. $410+041.244+03$

$3.418+041.239+03$

$3.426+041.237+03$

3. $434+041.242+03$

$3.441+041.254+03$

3. $445+041.268+03$

$3.449+041.314+03$

$3.453+041.471+03$

$3.457+04 \quad 4.595+03$

3. $461+046.956+03$

$3.465+046.900+03$

$3.469+046.917+03$

$3.473+046.902+03$

$3.477+046.875+03$

$3.481+046.862+03$

$3.485+046.830+03$

3. $489+046.794+03$

$3.493+046.756+03$

$3.497+046.723+03$

$3.501+046.681+03$

3. $505+046.650+03$

$3.509+046.619+03$

$3.513+046.589+03$

$3.517+046.555+03$

$3.521+046.532+03$

$3.525+046.502+03$

$3.529+046.472+03$

$3.533+046.453+03$

3. $537+046.425+03$

3. $541+046.403+03$

3. $545+046.386+03$

$3.549+046.358+03$

3. $553+046.340+03$

$3.557+046.317+03$

3. $561+046.294+03$

3. $565+046.278+03$

$3.569+046.258+03$

$3.573+046.232+03$

$3.577+046.213+03$

$3.581+046.203+03$

$3.585+046.174+03$

$3.589+046.151+03$

$3.593+046.139+03$ 
$3.594+046.129+03$

$3.598+046.106+03$

3. $601+046.091+03$

3. $606+046.064+03$

$3.610+046.050+03$

3. $650+045.873+03$

$3.690+045.684+03$

3. $730+045.494+03$

$3.734+045.478+03$

3. $738+045.465+03$

$3.742+045.446+03$

$3.746+045.433+03$

$3.750+045.422+03$

3. $790+045.251+03$

$3.830+045.108+03$

$3.870+04 \quad 4.966+03$

$3.883+044.914+03$

$3.887+044.912+03$

$3.891+04 \quad 4.892+03$

$3.895+04 \quad 4.904+03$

3. $899+044.880+03$

$3.903+044.850+03$

$3.907+04 \quad 4.835+03$

3. $911+04 \quad 4.821+03$

3. $915+04 \quad 4.824+03$

3. $919+044.803+03$

3. $923+044.791+03$

$3.927+044.773+03$

$3.931+044.765+03$

3. $935+044.746+03$

3. $939+044.738+03$

3. $943+044.720+03$

$3.947+04 \quad 4.712+03$

3. $951+04 \quad 4.703+03$

3. $955+044.683+03$

$3.959+044.683+03$

$3.963+044.658+03$

$3.967+044.646+03$

$3.971+044.633+03$

3. $975+04 \quad 4.627+03$

$3.979+044.615+03$

$3.983+044.591+03$

$3.987+044.601+03$

3. $991+044.576+03$

$3.995+044.575+03$

$3.999+044.541+03$

$4.003+044.539+03$

$4.007+04 \quad 4.537+03$

$4.011+04 \quad 4.515+03$

$4.015+04 \quad 4.507+03$

4. $019+044.488+03$

$4.023+044.480+03$

$4.027+044.469+03$

$4.031+044.458+03$

$4.035+04 \quad 4.451+03$

$4.039+044.431+03$

$4.043+044.428+03$

$4.047+04 \quad 4.421+03$

$4.051+044.413+03$

$4.055+04 \quad 4.391+03$

$4.059+04 \quad 4.371+03$
$3.595+046.123+03$

$3.599+046.104+03$

$3.603+046.081+03$

$3.607+046.070+03$

3. $620+045.994+03$

3. $660+045.815+03$

3. $700+045.638+03$

3. $731+045.498+03$

$3.735+045.473+03$

3. $739+045.461+03$

3. $743+045.452+03$

3. $747+045.422+03$

$3.760+045.380+03$

$3.800+045.223+03$

3. $840+045.075+03$

$3.880+044.933+03$

3. $884+044.922+03$

3. $888+044.906+03$

3. $892+04 \quad 4.895+03$

3. $896+04 \quad 4.879+03$

3. $900+04 \quad 4.861+03$

3. $904+044.843+03$

3. $908+044.842+03$

3. $912+044.819+03$

3. $916+044.816+03$

3. $920+044.799+03$

3. $924+044.788+03$

$3.928+04 \quad 4.773+03$

$3.932+04 \quad 4.761+03$

$3.936+04 \quad 4.749+03$

3. $940+044.742+03$

3. $944+04 \quad 4.727+03$

$3.948+04 \quad 4.711+03$

$3.952+044.691+03$

3. $956+04 \quad 4.684+03$

$3.960+044.668+03$

$3.964+044.656+03$

$3.968+04 \quad 4.658+03$

$3.972+044.629+03$

$3.976+04 \quad 4.627+03$

3. $980+044.611+03$

3. $984+04 \quad 4.603+03$

3. $988+044.591+03$

3. $992+044.578+03$

$3.996+04 \quad 4.563+03$

$4.000+04 \quad 4.554+03$

$4.004+04 \quad 4.534+03$

$4.008+044.531+03$

$4.012+044.522+03$

$4.016+04 \quad 4.510+03$

$4.020+04 \quad 4.495+03$

$4.024+04 \quad 4.468+03$

$4.028+04 \quad 4.464+03$

$4.032+04 \quad 4.447+03$

$4.036+04 \quad 4.444+03$

$4.040+04 \quad 4.445+03$

$4.044+04 \quad 4.429+03$

$4.048+044.403+03$

$4.052+04 \quad 4.396+03$

$4.056+04 \quad 4.388+03$

$4.060+044.376+03$
$3.596+046.113+03$

3. $600+046.098+03$

3. $604+046.085+03$

3. $608+046.060+03$

$3.630+045.959+03$

$3.670+045.763+03$

3. $710+045.582+03$

$3.732+045.483+03$

3. $736+045.472+03$

3. $740+045.465+03$

3. $744+045.445+03$

3. $748+045.429+03$

$3.770+045.340+03$

3. $810+045.190+03$

$3.850+045.039+03$

3. $881+044.927+03$

3. $885+04 \quad 4.911+03$

$3.889+044.905+03$

$3.893+044.896+03$

$3.897+044.876+03$

$3.901+044.869+03$

3. $905+04 \quad 4.850+03$

3. $909+04 \quad 4.843+03$

3. $913+044.821+03$

$3.917+044.805+03$

3. $921+044.800+03$

3. $925+044.791+03$

$3.929+044.771+03$

$3.933+044.769+03$

3. $937+044.750+03$

$3.941+044.737+03$

$3.945+044.721+03$

3. $949+044.705+03$

$3.953+044.700+03$

3. $957+044.675+03$

3. $961+044.659+03$

$3.965+04 \quad 4.653+03$

$3.969+044.646+03$

$3.973+04 \quad 4.631+03$

$3.977+044.622+03$

3. $981+044.599+03$

$3.985+044.594+03$

$3.989+044.589+03$

3. $993+044.568+03$

$3.957+044.558+03$

$4.001+044.540+03$

$4.005+044.523+03$

$4.009+04 \quad 4.529+03$

$4.013+044.511+03$

$4.017+044.504+03$

$4.021+04 \quad 4.492+03$

$4.025+044.481+03$

$4.029+044.458+03$

$4.033+04 \quad 4.445+03$

$4.037+04 \quad 4.441+03$

$4.041+044.434+03$

$4.045+04 \quad 4.422+03$

4. $049+044.406+03$

$4.053+044.383+03$

$4.057+044.380+03$
$3.597+046.109+03$

3. $601+046.097+03$

3. $605+046.070+03$

$3.609+046.047+03$

3. $640+045.896+03$

3. $680+045.728+03$

3. $720+045.547+03$

$3.733+045.489+03$

$3.737+045.469+03$

3. $741+045.451+03$

3. $745+045.434+03$

$3.749+045.423+03$

$3.780+045.302+03$

3. $820+045.144+03$

$3.860+045.008+03$

3. $882+044.927+03$

3. $886+044.912+03$

$3.890+044.909+03$

$3.894+044.880+03$

$3.898+04 \quad 4.876+03$

$3.902+044.854+03$

3. $906+044.846+03$

3. $910+044.839+03$

3. $914+044.826+03$

3. $918+044.808+03$

3. $922+044.792+03$

3. $926+044.780+03$

$3.930+044.759+03$

3. $934+044.754+03$

3. $938+044.740+03$

3. $942+044.722+03$

3. $946+044.713+03$

$3.950+044.701+03$

3. $954+044.697+03$

3. $958+044.679+03$

3. $962+044.669+03$

3. $966+044.655+03$

$3.970+04 \quad 4.647+03$

3. $974+04 \quad 4.624+03$

3. $978+04 \quad 4.617+03$

3. $982+044.606+03$

$3.986+044.600+03$

$3.990+044.578+03$

3. $994+044.556+03$

$3.998+044.560+03$

$4.002+044.545+03$

$4.006+044.542+03$

4. $010+04 \quad 4.525+03$

4. $014+04 \quad 4.514+03$

$4.018+044.500+03$

4. $022+04 \quad 4.486+03$

$4.026+04 \quad 4.472+03$

$4.030+04 \quad 4.458+03$

4. $034+04 \quad 4.438+03$

$4.038+04 \quad 4.438+03$

$4.042+044.428+03$

$4.046+04 \quad 4.417+03$

4. $050+044.398+03$

$4.054+044.403+03$

$4.058+044.386+03$ 
$\mathrm{Z}=68, \operatorname{Er}$

1. $221+055 \cdot 940+02$

$Z=72$, Hf

$1.390+045.246+04$

$5.950+049.781+02$

$Z=73, \mathrm{Ta}$

$1.487+035.512+05$

$5.000+031.658+05$

$8.000+03 \quad 4.996+04$

$1.350+045.041+04$

1. $700+04 \quad 2.790+04$

$3.500+04 \quad 4.443+03$

$\mathrm{Z}=73, \mathrm{Ta}$

$5.895+031.066+05$

$\mathrm{Z}=73, \mathrm{Ta}$

$6.400+038.746+04$

$\mathrm{Z}=73, \mathrm{Ta}$

$6.460+038.669+04$

$\mathrm{Z}=73, \mathrm{Ta}$

$8.118+03 \quad 4.777+04$

$\mathrm{Z}=73, \mathrm{Ta}$

1. $390+045.228+04$

$5.950+041.049+03$

$\mathrm{Z}=74, \mathrm{~W}$

$2.260+042.937+03$

$\mathrm{Z}=74, \mathrm{~W}$

$2.630+049.556+03$

$Z=75, \operatorname{Re}$

1. $780+043.046+04$

$Z=78, P t$

$1.000+031.469+06$

$4.000+033.624+05$

$6.000+031.328+05$

$9.500+03 \quad 4.117+04$

$1.100+042.790+04$

$2.000+04 \quad 2.339+04$

$4.000+04 \quad 3.867+03$

$\mathrm{Z}=78, \mathrm{Pt}$

$1.156+04 \quad 4.410+04$

$\mathrm{Z}=78, \mathrm{Pt}$

$5.300+041.996+03$

$1.220+051.012+03$

$\mathrm{Z}=78, \mathrm{Pt}$

$7.962+042.900+03$

$Z=78$, Pt

$1.098+051.290+03$

$\mathrm{eV}, \mathrm{b} /$ atom pairs

1. $365+05 \quad 4.490+02$

$\mathrm{eV}, \mathrm{b} / \mathrm{atom}$ pairs

$1.780+042.602+04 \quad 2.630+04$

5 pts

$9.632+03$

$\mathrm{eV}, \mathrm{b} / \mathrm{atom}$ pairs

22 pts

$1.500+03 \quad 5.251+05 \quad 3.000+03 \quad 5.380+05$

$5.415+03 \quad 1.416+05 \quad 5.947+03 \quad 1.081+05$

$8.398+03 \quad 4.410+04 \quad 9.000+03 \quad 3.632+04$

$1.400+04 \quad 4.605+04 \quad 1.450+04 \quad 4.248+04$

$2.000+04 \quad 1.881+04 \quad 2.494+04 \quad 1.069+04$

$4.000+04 \quad 3.067+03$

$\mathrm{eV}, \mathrm{b} / \mathrm{atom}$ pairs

$8.041+034.887+04$

eV, b/atom pairs

ev, b/atom pairs

$\mathrm{eV}, \mathrm{b} / \mathrm{atom}$ pairs

$\mathrm{eV}, \mathrm{b} / \mathrm{atom}$ pairs

$1.780+042.329+04 \quad 2.630+04 \quad 7.993+03$

ev, b/atom pairs

1 pts

3 pts

$\mathrm{eV}, \mathrm{b} / \mathrm{atom}$ pairs

$3.320+045.007+035.950+041.038+03$

$\mathrm{eV}, \mathrm{b} / \mathrm{atom}$ pairs

$2.630+041.024+04 \quad 3.320+04 \quad 5.505+03$

$\mathrm{eV}, \mathrm{b} /$ atom pairs

$1.487+036.970+05 \quad 1.500+036.834+05$

$5.000+032.089+05 \quad 5.415+03 \quad 1.752+05$

$8.000+03 \quad 6.404+04 \quad 8.398+03 \quad 5.613+04$

$9.962+03 \quad 3.663+04 \quad 1.000+04 \quad 3.605+04$

$1.450+04 \quad 5.403+04 \quad 1.500+04 \quad 5.030+04$

$2.494+041.351+04 \quad 3.000+04 \quad 8.373+03$

$\mathrm{eV}, \mathrm{b} / \mathrm{atom}$ pairs

$1.165+04 \quad 5.995+04 \quad 1.232+04$

3 pts

$\mathrm{eV}, \mathrm{b} / \mathrm{atom}$ pairs

$5.950+04 \quad 1.440+03 \quad 8.100+04 \quad 2.715+03$

$1.520+055.640+02$

eV, b/atom pairs

$8.100+04 \quad 2.740+03 \quad 8.091+04$

3 pts

eV, b/atom pairs
$2.782+03$

1 pt ref $=86$ De02 2

$4.000+032.851+05$

$6.000+031.040+05$

$9.500+03 \quad 3.136+04$

$1.500+04 \quad 4.025+04$

3. $000+046.525+03$

ref $=94 \mathrm{KeOI}$

ref $=93 \mathrm{KeO} 2$

ref $=93 \mathrm{KeO} 01$

ref $=93 \mathrm{Ke} 03$

ref $=95 \mathrm{Ta01}$

$3.320+04 \quad 4.447+03$

ref $=90$ Qu01

ref $=95 \mathrm{Ta} 01$

ref $=95 \mathrm{Ta} 01$

$5.950+041.135+03$

ref $=86$ De 02

$2.000+03 \quad 3.462+05$

$5.947+031.393+05$

9. $000+03 \quad 4.726+04$

$1.050+04 \quad 3.166+04$

1. $700+04 \quad 3.472+04$

$3.500+045.529+03$

ref $=88 \mathrm{PaO} 1$

ref $=87 \mathrm{MaO}$

$9.900+041.692+03$

ref $=87 \operatorname{Re} 01$

ref $=87 \mathrm{Sa0} 2$ 
$\mathrm{Z}=78$, Pt

1. $221+059.680+05$

$Z=79, \mathrm{Au}$

1. $000+03 \quad 1.522+06$

4. $000+033.806+05$

$6.000+031.399+05$

9. $500+034.352+04$

1. $100+042.971+04$

2. $000+04 \quad 2.457+04$

$4.000+04 \quad 4.159+03$

$\mathrm{Z}=79, \mathrm{Au}$

1. $192+04 \quad 4.229+04$

$Z=79, \mathrm{Au}$

$4.300+043.541+03$

$9.900+041.734+03$

$\mathrm{Z}=79$, Au

$7.962+047.200+02$

$\mathrm{Z}=82, \mathrm{~Pb}$

1. $487+038.916+05$

$5.000+032.577+05$

8. $000+037.946+04$

9. $962+03 \quad 4.517+04$

$1.150+043.075+04$

2. $000+042.921+04$

$4.000+04 \quad 4.885+03$

$\mathrm{Z}=82, \mathrm{~Pb}$

$5.893+031.662+05$

$\mathrm{Z}=82, \mathrm{~Pb}$

1. $343+04 \quad 4.693+04$

$5.920+041.585+03$

$\mathrm{Z}=82, \mathrm{~Pb}$

5. $240+042.257+03$

$\mathrm{Z}=82, \mathrm{~Pb}$

5. $300+042.485+03$

1. $220+051.164+03$

$\mathrm{Z}=82, \mathrm{~Pb}$

1. $236+051.142+03$

$\mathrm{Z}=82, \mathrm{~Pb}$

1. $452+057.569+02$

$\mathrm{Z}=82, \mathrm{~Pb}$

3. $200+051.169+02$

$\mathrm{Z}=83, \mathrm{Bi}$

$7.280+061.606+01$

$\mathrm{Z}=90, \mathrm{Th}$

$8.000+012.500+06$

$8.600+012.600+06$

$9.000+011.500-07$

$9.300+013.35+5$

eV, b/atom pairs

1. $365+057.260+02$

$\mathrm{eV}, \mathrm{b} / \mathrm{atom}$ pairs

25 pts

$1.487+03 \quad 7.220+05 \quad 1.500+03 \quad 7.021+05$

$5.000+03 \quad 2.199+05 \quad 5.415+03 \quad 1.822+05$

$8.000+03 \quad 6.082+04 \quad 8.398+03 \quad 5.938+04$

$9.962+03 \quad 3.872+04 \quad 1.000+04 \quad 3.649+04$

$1.150+04 \quad 2.636+04 \quad 1.500+04 \quad 5.271+04$

$2.494+04 \quad 1.437+04 \quad 3.000+04 \quad 8.937+03$

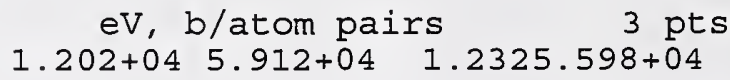

$\mathrm{eV}, \mathrm{b} / \mathrm{atom}$ pairs

7 pts

$5.300+04 \quad 2.064+03 \quad 5.950+04 \quad 1.512+03$

$1.220+051.040+03 \quad 1.520+05 \quad 6.020+02$

eV, b/atom pairs 3 pts

$8.100+042.870+03 \quad 8.091+04 \quad 2.530+03$

eV, b/atom pairs

25 pts

$1.500+03 \quad 8.658+05 \quad 2.000+03 \quad 4.561+05$

$5.415+032.140=05 \quad 5.947+03 \quad 1.697+05$

$8.398+03 \quad 6.997+04 \quad 9.000+03 \quad 5.855+04$

$1.000+04 \quad 4.458+04 \quad I .050+04 \quad 3.894+04$

$1.200+04 \quad 2.751+04 \quad 1.250+04 \quad 2.469+04$

$2.494+041.664+04 \quad 3.000+04 \quad 1.026+04$

eV, b/atom pairs

$1.337+045.100+04 \quad 1.744+04$

eV, b/atom pairs

$\begin{array}{llll}1.722+04 & 4.026+04 & 2.016+04 & 2.821+04\end{array}$

ev, b/atom pairs

1 pts

eV, b/atom pairs

6 pts

$5.950+041.718+03 \quad 8.100+047.660+02$ $1.520+056.980+02$

ev, b/atom pairs

1 pts

ev, b/atom pairs

1 pts

eV, b/atom pairs

1 pts

eV, b/atom pairs

$1 \mathrm{pt}$

eV, b/atom pairs

8. $200+012.300+06$

$8.700+012.500+06$

$9.050+01 \quad 1.850+07$

$9.400+013.500+06$
8. $400+012.200+06$

$8.800+013.000+06$

9. $100+011.300+07$

$9.500+014.500+06$ ref $=87 \mathrm{SaOI}$

ref $=86 \mathrm{De} 02$

$2.000+033.630+05$

$5.947+031.441+05$

$9.000+034.990+04$

1. $050+04 \quad 3.358+04$

1. $700+043.640+04$

$3.500+045.906+03$

ref $=88 \mathrm{PaOl}$

ref $=87 \mathrm{Ma01}$

8. $100+042.522+03$

ref $=87$ Re01

ref $=86$ De02

$4.000+034.376+05$

$6.000+031.649+05$

$9.500+035.105+04$

1. $100+043.454+04$

1. $700+04 \quad 4.242+04$

$3.500+046.904+03$

ref $=88 \mathrm{NaOl}$

2. $016+042.480+04$

ref $=84 \mathrm{NaO}$

$4.652+043.124+03$

ref $=89 \mathrm{Ke} 01$

ref $=87 \mathrm{MaO} 1$

$9.900+041.936+03$

ref $=92 \mathrm{Ja} 01$

ref $=93 \mathrm{JaO}$

ref $=92 \mathrm{JaO} 2$

ref $=84 \mathrm{Ye} 01$

ref $=87 \mathrm{De} 02$

$8.500+012.200+06$

$8.900+015.000+06$

9. $200+014.500+06$

$9.600+016.500+06$ 
$9.700+019.500+06$ 1. $010+023.400+07$

$1.050+01 \quad 3.950+07$

$1.090+022.800+07$

$1.130+02 \quad 2.100+07$

$1.170+021.700+07$

1. $210+021.450+07$

1. $250+021.270+07$

1. $290+021.120+07$

1. $330+021.000+07$

$1.370+028.000+06$

1. $410+026.800+06$

1. $450+025.500+06$

1. $490+024.920+06$

1. $530+024.000+06$

$1.570+02 \quad 3.300+06$

1. $700+021.800+06$

$\mathrm{Z}=92, \mathrm{U}$

$8.000+015.000+06$

9. $600+01 \quad 3.400+06$

1. $020+02 \quad 1.300+07$

$1.080+021.600+07$

1. $160+025.300+07$

1. $240+02 \quad 2.700+07$

1. $320+021.750+07$

1. $400+021.250+07$

$1.480+021.000+07$

1. $560+028.000+06$

1. $640+026.000+06$

1. $720+024.800+06$

1. $800+024.000+06$

$\mathrm{Z}=92, \mathrm{U}$

1. $487+03 \quad 1.399+06$

$5.947+032.440+05$

1. $000+047.145+04$

3. $000+041.612+04$
$9.800+011.350+07$

$1.020+024.000+07$

$1.060+023.600+07$

1. $100+022.550+07$

$1.140+022.050+07$

1. $180+021.600+07$

1. $220+021.400+07$

1. $260+021.220+07$

1. $300+021.100+07$

1. $340+029.500+06$

1. $380+027.600+06$

1. $420+026.300+06$

1. $460+025.200+06$

1. $500+024.600+06$

1. $540+023.800+06$

1. $580+023.000+06$

1. $750+021.000+06$

eV, b/atom pairs

$8.500+013.000+06$

$9.800+017.000+06$

1. $040+028.000+06$

1. $100+022.650+07$

1. $180+024.700+07$

1. $260+02 \quad 2.450+07$

1. $340+021.600+07$

1. $420+021.200+07$

$1.500+029.700+06$

$1.580+027.000+06$

1. $660+025.600+06$

1. $740+024.300+06$

$9.900+01 \quad 1.850+07$

$1.030+024.400+07$

$1.070+02 \quad 3.300+07$

1. $110+022.400+07$

1. $150+021.900+07$

$1.190+021.550+07$

$1.230+021.360+07$

$1.270+021.180+07$

1. $310+021.050+07$

$1.350+028.600+06$

1. $390+027.500+06$

1. $430+026.200+06$

$1,470+025.000+06$

1. $510+024.200+06$

1. $550+023.600+06$

1. $600+022.800+06$

1. $800+025.000+05$

1. $000+022.750+07$

1. $040+02 \quad 4.400+07$

$1.080+022.950+07$

1. $120+02 \quad 2.200+07$

1. $160+021.800+07$

1. $200+02 \quad 1.500+07$

1. $240+021.300+07$

1. $280+021.150+07$

1. $320+021.000+07$

1. $360+028.300+06$

$1.400+026.900+06$

1. $440+026.000+06$

1. $480+024.950+06$

1. $520+024.000+06$

1. $560+02 \quad 3.500+06$

1. $650+022.300+06$

ref $=87 \mathrm{De} 01$

9.500+01 3.000+06

1. $005+021.650+07$

1. $060+021.000+07$

1. $140+024.950+07$

1. $220+02 \quad 3.250+07$

1. $300+02 \quad 2.000+07$

1. $380+021.300+07$

$1.460+021.050+07$

1. $540+028.500+06$

$1.620+026.000+06$

$1.700+025.000+06$

$1.780+024.000+06$

$\mathrm{eV}, \mathrm{b} /$ atom pairs

$1.500+031.367+06$

$6.000+032.394+05$

1. $500+042.524+04$

3. $500+041.070+04$
$.000+037.517+05$

$8.000+031.197+05$

1. $700+041.791+04$

$4.000+047.702+03$ ref $=86 \mathrm{De} 02$

3. $000+032.896+05$

$9.962+037.212+04$

$2.494+042.576+04$ 
Table 2. Summary of Photon Cross Section Data Extracted (47 Papers).

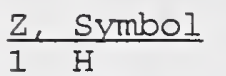

$2 \mathrm{He}$

$3 \mathrm{Li}$

$4 \mathrm{Be}$

$6 \mathrm{C}$

$7 \mathrm{~N}$

80

$11 \mathrm{Na}$

$12 \mathrm{Mg}$

$13 \mathrm{AI}$

$14 \mathrm{Si}$

18 Ar

$22 \mathrm{Ti}$

$23 \mathrm{~V}$

$24 \mathrm{Cr}$

$26 \mathrm{Fe}$

$27 \mathrm{Co}$

$28 \mathrm{Ni}$

$29 \mathrm{Cu}$

$30 \mathrm{Zn}$

$36 \mathrm{Kr}$

$39 Y$

$40 \mathrm{Zr}$

$41 \mathrm{Nb}$

$42 \mathrm{MO}$

$46 \mathrm{Pd}$

$47 \mathrm{Ag}$

$48 \mathrm{Cd}$

49 In

$50 \mathrm{Sn}$

53 I

$54 \mathrm{Xe}$

68 Er

72 Hf

$73 \mathrm{Ta}$

$74 \mathrm{~W}$

$75 \mathrm{Re}$

$78 \mathrm{Pt}$

$79 \mathrm{Au}$

$82 \mathrm{~Pb}$

$83 \mathrm{Bi}$

$90 \mathrm{Th}$

$92 \mathrm{U}$

\section{Number of papers with data}

3

2

1

3

4

4

1

3

14

4

2

5

1

3

6

1

16

3

2

1

1

1

3

4

7

5

3

7

1

3

1

1

6

2

1

6

4

1

1

2

Total Data Points Extracted: $\frac{\text { Data points }}{20}$

118

8

15

44

51

117

21

231

590

182

22

131

19

345

96

20

403

176

28

53

35

5

7

17

27

18

63

74

9
468

2

32

4

4
40

38

44

1

83

64

3735 

